\title{
Cooperative Search of Multiple Unknown Transient Radio Sources Using Multiple Paired Mobile Robots
}

\author{
Chang-Young Kim, Member, IEEE, Dezhen Song, Senior Member, IEEE, Yiliang Xu, Member, IEEE, \\ Jingang Yi, Senior Member, IEEE, and Xinyu Wu
}

\begin{abstract}
We develop a localization method to enable a team of mobile robots to search for multiple unknown transient radio sources. Because of signal source anonymity, short transmission durations, and dynamic transmission patterns, robots cannot treat the radio sources as continuous radio beacons. Moreover, robots do not know the source transmission power and have limited sensing ranges. To cope with these challenges, we pair up robots and develop a cooperative sensing model using signal strength ratios from the paired robots. We formally prove that the joint conditional posterior probability of source locations for the $m$-robot team can be obtained by combining the pairwise joint posterior probabilities, which can be derived from signal strength ratios. Moreover, we propose a pairwise ridge walking algorithm (PRWA) to coordinate the robot pairs based on the clustering of high-probability regions and the minimization of local Shannon entropy. We have implemented and validated the algorithm under both the hardware-driven simulation and physical experiments. Experimental results show that the PRWA-based localization scheme consistently outperforms the other four heuristics.
\end{abstract}

Index Terms-Radio localization, robot motion planning, unknown sensor network.

\section{INTRODUCTION}

I MAGINE that a team of mobile robots is searching for a sensor network deployed by enemies (see Fig. 1). The robots have little information about the sensor network, except for the fact that the sensor nodes emit short radio signals from time to time. Without the knowledge of the network configuration and packet structure, localizing each node is difficult because of signal source anonymity, short transmission durations, and dynamic/intermittent transmission patterns. The robots can only rely on the received signal strength (RSS) from intercepted sig-

Manuscript received June 21, 2013; revised December 31, 2013; accepted June 18, 2014. Date of publication July 16, 2014; date of current version September 30, 2014. This paper was recommended for publication by Associate Editor V. Isler and Editor D. Fox upon evaluation of this reviewers' comments. This work was supported in part by the National Science Foundation under IIS-1318638 and by a grant from Shenzhen Science and Technology Project [Reference No. ZDS Y20120617113312191]. This paper has supplementary downloadable material available at http://ieeexplore.ieee.org.

C.-Y. Kim is with Neato Robotics, Newark, CA 94560 USA (e-mail: kcyoung 98@gmail.com).

D. Song is with the Department of Computer Science and Engineering, Texas A\&M University, College Station, TX 77843 USA (e-mail: dzsong@ cs.tamu.edu).

Y. Xu is with Kitware, Inc., Clifton Park, NY 12065 USA (e-mail: yiliang. xu@kitware.com).

J. Yi is with the Department of Mechanical and Aerospace Engineering, Rutgers University, Piscataway, NJ 08854 USA (e-mail: jgyi@ rutgers.edu).

$\mathrm{X}$. $\mathrm{Wu}$ is with the Shenzhen Institute of Advanced Technology, Chinese Academy of Science, Shenzhen 518055, China (e-mail: xy.wu@ siat.ac.cn).

Color versions of one or more of the figures in this paper are available online at http://ieeexplore.ieee.org.

Digital Object Identifier 10.1109/TRO.2014.2333097

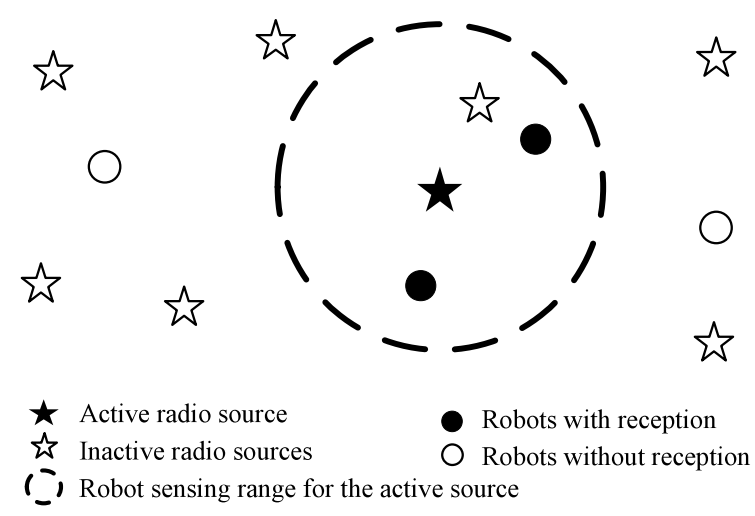

Fig. 1. Example of the localization scenario.

nals. However, the transmission power of the radio sources is unknown and may vary from time to time. A new method is needed for this multisource localization problem that is coupled with issues in signal correspondence, variable source transmission power, and robot sensing range limits.

Here, we present a search method to enable a team of mobile robots to localize multiple unknown and transient radio sources. The contributions of this paper are twofold. First, we formally prove that the joint conditional posterior probability of source locations given RSS readings from the $m$-robot team can be obtained by combining the joint conditional posterior probabilities of all pairs. The pairwise joint conditional posterior probabilities are derived from RSS ratios. This result allows the approach to handle unknown and variable source signal transmission power. The new sensing model is combined with the spatiotemporal probability occupancy grid (SPOG) to address signal correspondence issue. SPOG tracks the source location distributions and signal transmission frequency. Second, we propose a pairwise ridge walking algorithm (PRWA) to coordinate robot pairs based on the clustering of high-probability regions and the minimization of local Shannon entropy. We have implemented and validated the algorithm under a hardware-driven simulation and physical experiments. In the experiments, we compare PRWA with four heuristic methods: pairwise patrol, pairwise random walk, regular patrol, and regular random walk. Results show that the PRWA-based localization consistently outperforms the other four heuristics in all settings.

The remainder of this paper is organized as follows. We first review the related work in Section II. We introduce the localization system design and formulate two problems in Section III, which include the sensing problem addressed in Section IV and the robot motion planning problem addressed in Section V. 
Section VI summarizes the development into two algorithms. Both algorithms are validated in experiments in Section VII before we conclude the paper in Section VIII.

\section{RELATED WORK}

The recent development of radio frequency-based localization can be viewed as the localization of "friendly" radio sources because researchers either assume that an individual radio source continuously transmits radio signals (similar to a lighthouse) [1]-[4] or assume that robots/receivers are a part of the network and understand the detailed packet information [5]-[7]. However, protocol and network information is not always available for an unknown network. Radio signals are very short and cannot be easily associated with their transmitters. When signal correspondence cannot be established between different listening time and locations, most existing methods would have difficulty to handle the problem.

When signal sources are not cooperative, RSS readings are the primary information for localization because RSS attenuates over distance. Since signal transmission power at the source is not available, ratios between RSS readings from dislocated listeners have been proven to be effective [8]-[10]. Li et al. [11] show that at least four robots are needed at the same moment in order to localize a single source with unknown transmission power. Some other approaches use antenna arrays to obtain bearing readings. Kim and Chong [12] show how to find a radio source using two antennas with different polarizations. These approaches focus on single source localization and, hence, are not concerned with the signal correspondence issue.

Localization of multiple radio sources with multiple robots is structurally similar to multirobot simultaneous localization and mapping (SLAM) problem [13]-[15]. Although both SLAM approaches and our approach may share a Bayesian framework, SLAM assumes static environment while networked radio sources are highly dynamic because of ever changing signal transmission patterns. Another major difference is that the sensing of the environment can be done individually by robots in exploration [16] in SLAM, while our robots have to cooperatively listen and infer signals with unknown/variable source transmission power. Depth to signal sources cannot be obtained by an individual robot. Our new framework is designed to handle these new challenges.

In the sensing part of our problem, we partition the open 2-D space into equalized grid cells. This grid-based approach extends existing framework on occupancy grid (OG) maps [17], [18]. OG is a spatial probabilistic sensor model and has been proved to be an elegant representation of the sensor coverage for mobile robot applications [19]. Recent developments on OG include multisensor fusion [20], an inverse sensor model, and a forward sensor model. The existing OG-based methods use the spatial probabilistic representation to describe sensing uncertainty and cannot deal with time-variant environments. In this study, we extend the OG methods into the temporal dimension and allow multiple robots to collaboratively share/update OG to deal with the dynamic characteristics of the transient radio transmissions.
In the planning part of our problem, we purposefully partition robots into pairs. This can be viewed as a special robot formation. Mobile robot formation control has been a popular research area in recent years [21], [22]. While most of distributed mobile robots work concerns the control aspect of the problem, our approach is mainly focused on the sensing-constraint planning rather that the low-level control. In the existing literature, the Roumeliotis et al. works on range-based localization[23]-[25] are closely related. They estimate robot pose and relative position using range readings in a robot network. In a way, our approach is also range based because we use RSS readings to derive range data. The Huynh et al. work on persistent patrol [26] also concerns sensing range constraints with a prior distribution of targets in a 2-D space. Our work is also inspired by Bhadauria et al.'s [27] recent work where robot motions are abstracted to travel salesperson problem tours to facilitate coordination and planning. Although these works share the characteristics of being sensing-constraint planning, the unique issue in our problem is that the individual robot in our settings cannot obtain range readings without assistance of other robots and the aforementioned signal correspondence issue.

Realizing that localizing unknown transient radio sources is an important new problem, our group studies the problem under different setups and constraints. First, we assume a carrier sense multiple access-based protocol is used among networked radio sources [28], [29] which allows us to develop a particle filter-based approach. Then, we relax the assumption and develop a protocol-independent localization scheme using an SPOG [30], [31]. Our recent works [32]-[34] find that teamed robots are more efficient than a single robot when the target is transient under the same sensing coverage. A new decentralized framework is presented in [34]. That result shifts our attention to the multirobot-based approach in this paper. This paper significantly extends its conference version [35] by adding system design, algorithms, and physical experiment results.

\section{System ARChitecture AND PRoblem Definition}

\section{A. System Architecture}

Fig. 2 illustrates the system diagram for coordinating a threerobot team to search for unknown radio sources. Both robots and radio sources reside in a 2-D Euclidean space. The whole system can be divided by the horizontal dashed line into two parts: the sensing part and the planning part.

The sensing part is triggered by radio signal receptions. All robots are synchronized listeners. Once a radio signal is detected, some robots may have receptions, while others do not because of reception range limit. Each robot provides an initial estimation of the radio source distribution as a function of an unknown source power level using its antenna model. The system uses a pairwise sensing technique to remove the dependence on the unknown source power level by deriving the signal source distribution as a function of the RSS ratio from each robot pair. The pairwise sensing technique examines all pair combinations except pairs without readings by either robot. Then, the sensor fusion aggregates the outputs of pairwise sensing modules and updates SPOG. 


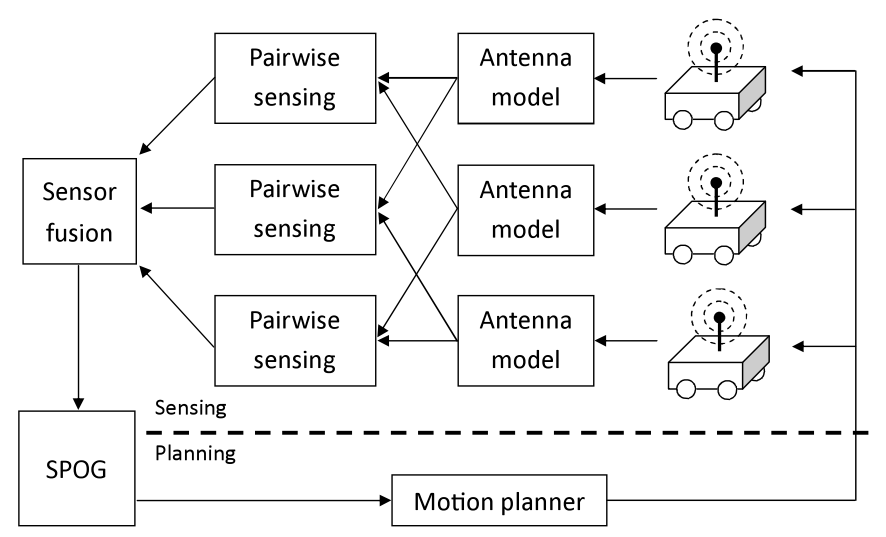

Fig. 2. System diagram for a three-robot case. In the sensing part, the pairwise sensing modules exams readings from any two robots if at least one of the two robots has an RSS reading. Pairs without readings are ignored as explained later. For an $m$-robot case, there can be as many as $m(m-1) / 2$ pairwise sensing modules.

SPOG is a Bayesian framework tracking the transmitter locations and transmission rates. SPOG can be viewed as a collective belief function for all radio sources. Based on the SPOG, the motion planner actively pairs up robots and allocates the paired robot teams to disjointed subregions with high probability to increase the searching efficiency. Each paired robot team patrols a subregion with its intrapair distance determined by minimizing local Shannon entropy over the subregion. Note that the paired robot team is not to be confused with the pairs in the sensing part. The pairs in the sensing part refers to any two robots in the field, while the paired robot team in the motion planning refers to two robots that are close to each other, as assigned by the motion planner.

The system design implies the following assumptions.

1) Each robot is equipped with an omnidirectional antenna with a limited sensing range.

2) All robots are coordinated using a centralized control.

3) The unknown network traffic is light, and each target radio transmission is short, which are the typical characteristics of a low-power sensor network.

4) Transmission powers of radio sources are unknown to the robots and may change from time to time. However, locations of radio sources do not change.

\section{B. Spatiotemporal Probability Occupancy Grid}

As shown in Fig. 2, SPOG bridges the sensing part with the planning part. It is originally proposed in our previous work [31] to handle a single-robot case. To avoid overlap, here, we only show how to extend SPOG to the high-dimensional case for the multiple robot team by skipping the details of SPOG.

SPOG partitions the searching region into small and equalsized grid cells. Define $i \in \mathcal{N}$ as the cell index variable, where $\mathcal{N}:=\{1, \ldots, n\}$ is the grid cell index set, and $n$ is the total number of cells. SPOG tracks two types of probabilistic events: $C_{i}$ represents the event that cell $i$ contains a radio source, and $C_{i}^{1}$ represents the event that cell $i$ is the active source when a transmission is detected. Define $P(C)$ as the probability for event
C. $P\left(C_{i}\right)$ and $P\left(C_{i}^{1}\right)$ characterize spatiotemporal behaviors of transient radio sources. Note that we ignore collision cases because robots have an RSS reading as soon as the transmission is initiated and the probability of two or more transmissions initiated at the exact same moment is negligible in a light traffic network.

Let $l \in \mathcal{M}:=\{1, \ldots, m\}$ be the robot index variable, where $m$ is the total number of robots, and $\mathcal{M}$ is the robot index set. $m$ is always an even natural number in our problem. Discrete time $k$ refers to each moment when a transmission is detected by robots. Let the discrete random variable $\tilde{Z}_{l}^{k} \in \mathbb{N}$ be the RSS reading (from a discrete receiver) of the $l$ th robot at time $k$. Define $\tilde{\mathbf{Z}}^{k}=\left[\tilde{Z}_{1}^{k}, \ldots, \tilde{Z}_{m}^{k}\right]^{T}$ as a discrete random vector of all the RSS readings at time $k$, and let $\tilde{\mathbf{z}}^{k}:=\left[\tilde{z}_{1}^{k}, \ldots, \tilde{z}_{m}^{k}\right]^{T}$ be corresponding values. As a convention, we use lower cases of random variables or vectors to denote their values.

At time $k$, event $\tilde{\mathbf{Z}}^{k}=\tilde{\mathbf{z}}^{k}$ is perceived by robots. The posterior probability $P\left(C_{i} \mid \tilde{\mathbf{Z}}^{k}=\tilde{\mathbf{z}}^{k}\right)$ over the grid needs to be updated. According to [31], this is actually a nested multivariate Bayesian process

$$
\begin{aligned}
& P\left(C_{i} \mid \tilde{\mathbf{Z}}^{k}=\tilde{\mathbf{z}}^{k}\right) \\
& =\frac{\left(\begin{array}{c}
P\left(\tilde{\mathbf{Z}}^{k}=\tilde{\mathbf{z}}^{k} \mid C_{i}^{1}\right) P\left(C_{i}^{1}\right)+ \\
P\left(C_{i}\right) \sum_{s \neq i, s \in I} P\left(\tilde{\mathbf{Z}}^{k}=\tilde{\mathbf{z}}^{k} \mid C_{s}^{1}\right) P\left(C_{s}^{1}\right)
\end{array}\right)}{\sum_{i \in I} P\left(\tilde{\mathbf{Z}}^{k}=\tilde{\mathbf{z}}^{k} \mid C_{i}^{1}\right) P\left(C_{i}^{1}\right)} \\
& P\left(C_{i}^{1} \mid \tilde{\mathbf{Z}}^{k}=\tilde{\mathbf{z}}^{k}\right) \\
& =\frac{P\left(\tilde{\mathbf{Z}}^{k}=\tilde{\mathbf{z}}^{k} \mid C_{i}^{1}\right) P\left(C_{i}^{1}\right)}{\sum_{i \in I} P\left(\tilde{\mathbf{Z}}^{k}=\tilde{\mathbf{z}}^{k} \mid C_{i}^{1}\right) P\left(C_{i}^{1}\right)}
\end{aligned}
$$

where $P\left(\tilde{\mathbf{Z}}^{k}=\tilde{\mathbf{z}}^{k} \mid C_{i}^{1}\right)$ is the sensing model. Equations (1) and (2) can be easily modified to an incremental conditional format for recursive update [30]. As more RSS readings enter the system over time, $P\left(C_{i} \mid \tilde{\mathbf{Z}}^{k}=\tilde{\mathbf{z}}^{k}\right)$ converges and allows robots to localize each radio source.

\section{Problem Formulation}

Again, we extend the problem definition in [31]. For completeness, we reiterate it here. To utilize the Bayesian framework, we need to derive a sensing model first.

Definition 1 (Sensing Problem): Derive $P\left(\tilde{\mathbf{Z}}^{k}=\tilde{\mathbf{z}}^{k} \mid C_{i}^{1}\right)$ for present time $k$ when a new RSS reading is received.

Once $P\left(\tilde{\mathbf{Z}}^{k}=\tilde{\mathbf{z}}^{k} \mid C_{i}^{1}\right)$ is obtained, we can use (1) and (2) to compute posterior sensor location distribution $P\left(C_{i} \mid \tilde{\mathbf{Z}}^{k}=\tilde{\mathbf{z}}^{k}\right)$. The SPOG gets updated for each perceived radio transmission.

Based on SPOG, the motion planner's task can be characterized as the following problem.

Definition 2 (Planning Problem): Given the updated $P\left(C_{i}\right.$ $\left.\tilde{\mathbf{Z}}^{k}=\tilde{\mathbf{z}}^{k}\right)$, plan trajectories for each robot at the beginning of each planning period.

Despite the similarity in problem structure with [31], solving the two problems is much harder for this multirobot multisource case. We start with the sensing problem first in Section IV. 


\section{SENSING MODEL}

The sensing model that computes $P\left(\tilde{\mathbf{Z}}^{k}=\tilde{\mathbf{z}}^{k} \mid C_{i}^{1}\right)$ is very complex. It is a joint conditional distribution of an $m$-dimensional random vector. To derive the conditional probability, we model the signal transmission uncertainty for rangelimited antenna, derive a pairwise sensing model based on signal strength ratio to remove the dependence on source transmission power, and propose a sensing fusion scheme to aggregate the output of all pairs to obtain the high-order model $P\left(\tilde{\mathbf{Z}}^{k}=\tilde{\mathbf{z}}^{k} \mid C_{i}^{1}\right)$. Actually, these three tasks correspond to the three functional blocks (i.e., antenna model, pairwise sensing, and sensor fusion) in the sensing part of Fig. 2. For simplicity, the time superscript $k$ is dropped in this section as all values correspond to present time $k$. Thus, $P\left(\tilde{\mathbf{Z}}^{k}=\tilde{\mathbf{z}}^{k} \mid C_{i}^{1}\right)$ becomes $P\left(\tilde{\mathbf{Z}}=\tilde{\mathbf{z}} \mid C_{i}^{1}\right)$.

\section{A. Antenna Model}

The purpose of the antenna model is to convert the RSS to the signal source location distribution. For a robot equipped with an omnidirectional antenna, the distance to the active radio source and source transmission power largely determine the RSS. Assume the active radio source is located at the center of the cell $i$. Let $\mathbf{x}_{\mathbf{i}}=\left[\mathbf{x}_{\mathbf{i}}, \mathbf{y}_{\mathbf{i}}\right]^{\mathbf{T}}$ and $\mathbf{x}_{\mathbf{l}}=\left[\mathbf{x}_{\mathbf{l}}, \mathbf{y}_{\mathbf{l}}\right]^{\mathbf{T}}$ be the center location of cell $i$ and the location of robot $l$, respectively, when the transmission is sensed. Define $d_{l i}=\left\|\mathbf{x}_{\mathbf{l}}-\mathbf{x}_{\mathbf{i}}\right\|$ as the Euclidean distance between $\mathbf{x}_{\mathbf{l}}$ and $\mathbf{x}_{\mathbf{i}}$. Following the signal propagation model [36], the expected RSS of robot $l$ is denoted as $\psi_{l}$ and measured in units of $\mathrm{dBm}$

$$
\psi_{l}=w_{i}-10 \beta \log _{10}\left(d_{l i}\right)
$$

where source power level $w_{i}$ is unknown, and $\beta$ is the signal decay factor.

An RSS level is not a constant but a continuous random variable because of uncertainties in transmissions. Assuming the robot radio listener has an infinite resolution, its RSS would be a continuous random variable $Z_{l}$ for robot $l$. Moreover, robots can only detect the transmission signal if an active radio source is located in their sensing ranges, each of which is determined by an RSS threshold denoted by $\zeta$. To characterize sensing range limit and background noises in sensing, we have

$$
Z_{l}=\mu_{l}+\omega_{l}, \quad \text { where } \mu_{l}=\left\{\begin{array}{l}
\psi_{l}, \text { if } \tilde{z}_{l}>\zeta \\
\zeta, \text { otherwise }
\end{array}\right.
$$

where $\omega_{l}$ follows the independent and identically distributed (i.i.d.) Gaussian with zero mean and a variance of $\sigma^{2}$. Note that $\beta$ in (3) and $\sigma^{2}$ can be obtained by calibration. Therefore, the probability density function (PDF) of $Z_{l} \mid C_{i}^{1}$ is $f_{Z_{l} \mid C_{i}^{1}}\left(z_{l}\right)=\operatorname{Bel}\left(\mu_{p}, \sigma^{2}\right)$, where $\operatorname{Bel}\left(\mu_{p}, \sigma^{2}\right)$ is the Gaussian PDF. As a convention, the subscript of $f(\cdot)$ is the corresponding random variable of the PDF function.

Remark 1: In practice, antennas often suffer from imprecise radiation patterns and unknown environmental reflections. We can increase $\sigma$ to capture the increased level of uncertainty. For simplicity, we assume omnidirectional radiation pattern in the analysis, which assumes a perfectly round radiation patterns. Actually, a far-field radiation pattern of an imprecise omnidirectional antenna can always be approximated by an ellipsoid.
In such a case, our method may lose some efficiency and accuracy but is still able to find the sensor location. If available, time of flight measurements can be used instead of RSS to reduce $\sigma$.

Actually, the RSS reading $\tilde{Z}_{l}$ is an integer because of receiver hardware limit. As a convention, we use $\tilde{a}$ to indicate the integer value of continuous variable $a$. Define $\mathcal{I}_{l}$ as an RSS interval

$$
\mathcal{I}_{l}=\left(\tilde{z}_{l}-0.5, \tilde{z}_{l}+0.5\right] \subset \mathbb{R} .
$$

Thus, we have the relationship between $\tilde{Z}_{l}$ and $Z_{l}$ given $C_{i}^{1}$

$$
P\left(\tilde{Z}_{l}=\tilde{z}_{l} \mid C_{i}^{1}\right)=P\left(Z_{l} \in \mathcal{I}_{l} \mid C_{i}^{1}\right)=\int_{z_{l} \in \mathcal{I}_{l}} f_{Z_{l} \mid C_{i}^{1}}\left(z_{l}\right) d z_{l} .
$$

This is actually the sensing model when there is only one robot. Since this model relies on the unknown source power level $w_{i}$, it is not a viable sensing model but provides a foundation for the next step.

\section{B. Transmission Power Independent Pairwise Sensing}

To remove the dependence on the source power level, we use signal ratio from a dislocated antenna/robot pair. This process is named as pairwise sensing. For $m$-robots in the field, we need to examine all $\left(\begin{array}{c}m \\ 2\end{array}\right)=\frac{m(m-1)}{2}$ pair combinations.

For a robot pair $(p, q), p \neq q$, recall the possible RSS readings form sets $\mathcal{I}_{p}$ and $\mathcal{I}_{q}$ as defined in (5), respectively. According to our convention, $P\left(Z_{p} \in \mathcal{I}_{p}, Z_{q} \in \mathcal{I}_{q} \mid C_{i}^{1}\right)$ is a pairwise conditional probability given $C_{i}^{1}$. We are now ready to show that $P\left(Z_{p} \in \mathcal{I}_{p}, Z_{q} \in \mathcal{I}_{q} \mid C_{i}^{1}\right)$ can be obtained from its RSS ratio regardless of source transmission power levels.

Define $Z_{p-q}:=Z_{p}-Z_{q}$, and let $\mathcal{I}_{p-q}=\left(\tilde{z}_{p}-\tilde{z}_{q}-\right.$ $\left.1, \tilde{z}_{p}-\tilde{z}_{q}+1\right] \subset \mathbb{R}$ be the interval of $Z_{p-q}$ values. $P\left(Z_{p-q} \in\right.$ $\left.\mathcal{I}_{p-q} \mid C_{i}^{1}\right)$ denotes the probability of pairwise difference given $C_{i}^{1}$. We have the following lemma with its proof in Appendix A.

\section{Lemma 1:}

$$
P\left(Z_{p} \in \mathcal{I}_{p}, Z_{q} \in \mathcal{I}_{q} \mid C_{i}^{1}\right)=\frac{1}{\eta_{p q}} P\left(Z_{p-q} \in \mathcal{I}_{p-q} \mid C_{i}^{1}\right)
$$

where $\eta_{p q}$ is the normalizing factor.

It is worth noting that, since the RSS readings are in log scale, the difference between the two readings $Z_{p-q}$ actually means an RSS ratio which does not depend on source transmission power levels. Computing $P\left(Z_{p-q} \in \mathcal{I}_{p-q} \mid C_{i}^{1}\right)$ is nontrivial because some of robots may not have readings because of limited sensing ranges. Based on (4), the robot index set $\mathcal{M}$ is partitioned into two disjoint sets $\mathcal{M}=\mathcal{M}_{1} \cup \mathcal{M}_{0}$, which correspond to the sets of robots with and without receptions, respectively. As a result, we have three types of pairs: no detection for either robot, single detection, and dual detection. Define $\mathcal{E}$ as the set for all possible pairs, which consists of three disjoint subsets $\mathcal{E}=\mathcal{E}_{11} \cup \mathcal{E}_{10} \cup \mathcal{E}_{00}$, where

$$
\begin{aligned}
& \mathcal{E}_{11}=\left\{(p, q) \mid p<q, p \in \mathcal{M}_{1}, q \in \mathcal{M}_{1}\right\} \\
& \mathcal{E}_{10}=\left\{(p, q) \mid p \in \mathcal{M}_{1}, q \in \mathcal{M}_{0}\right\} \\
& \mathcal{E}_{00}=\left\{(p, q) \mid p<q, p \in \mathcal{M}_{0}, q \in \mathcal{M}_{0}\right\} .
\end{aligned}
$$


Define $Z_{p-q}^{11}, Z_{p-q}^{10}$, and $Z_{p-q}^{00}$ as the sensor readings of the robot pair $(p, q)$ corresponding to components of $\mathcal{E}_{11}, \mathcal{E}_{10}$, and $\mathcal{E}_{00}$, respectively. $Z_{p-q}$ in (7) will be one of these three types. We now focus on deriving $P\left(Z_{p-q}^{11} \in \mathcal{I}_{p-q} \mid C_{i}^{1}\right), P\left(Z_{p-q}^{10} \in\right.$ $\left.\mathcal{I}_{p-q} \mid C_{i}^{1}\right)$, and $P\left(Z_{p-q}^{00} \in \mathcal{I}_{p-q} \mid C_{i}^{1}\right)$.

Let us compute $P\left(Z_{p-q}^{11} \in \mathcal{I}_{p-q} \mid C_{i}^{1}\right)$ first. Recall that $d_{q i}$ and $d_{p i}$ refer to the distance from robots $q$ and $p$ to the center of cell $i$, respectively. From (3) and (4), the mean value $\left(\mu_{p}-\mu_{q}\right)$ of $Z_{p-q}^{11}$ becomes

$$
\mu_{p}-\mu_{q}=\psi_{p}-\psi_{q}=10 \beta \log _{10} \frac{d_{q i}}{d_{p i}}
$$

and the PDF of $Z_{p-q}^{11} \mid C_{i}^{1}$ is

$$
f_{Z_{p-q}^{11} \mid C_{i}^{1}}\left(z_{p-q}^{11}\right)=\operatorname{Bel}\left(10 \beta \log _{10} \frac{d_{q i}}{d_{p i}}, 2 \sigma^{2}\right) .
$$

Thus, we have the following lemma.

Lemma 2:

$$
\begin{aligned}
P\left(Z_{p-q}^{11} \in \mathcal{I}_{p-q} \mid C_{i}^{1}\right)= & \int_{\tilde{z}_{p}-\tilde{z}_{q}-1}^{\tilde{z}_{p}-\tilde{z}_{q}+1} f_{Z_{p-q}^{11} \mid C_{i}^{1}}(z) d z \\
= & {\left[\mathrm{F}_{Z_{p-q}^{11} \mid C_{i}^{1}}\left(\tilde{z}_{p}-\tilde{z}_{q}+1\right)\right.} \\
& \left.-\mathrm{F}_{Z_{p-q}^{11} \mid C_{i}^{1}}\left(\tilde{z}_{p}-\tilde{z}_{q}-1\right)\right]
\end{aligned}
$$

where $\mathrm{F}_{Z_{p-q}^{11} \mid C_{i}^{1}}(\cdot)$ is the cumulative distribution function of $f_{Z_{p-q}^{11} \mid C_{i}^{1}}(\cdot)$.

To facilitate the understanding of the dual detection case, Fig. 3(a) shows an example to illustrate the corresponding posterior probability $P\left(C_{i}^{1} \mid Z_{p-q}^{11} \in \mathcal{I}_{p-q}\right)$. Note that what is in the figure is not $P\left(Z_{p-q}^{11} \in \mathcal{I}_{p-q} \mid C_{i}^{1}\right)$ that we just computed because the posterior spatial distribution $P\left(C_{i}^{1} \mid Z_{p-q}^{11} \in \mathcal{I}_{p-q}\right)$ is what we are actually interested in. We want to examine how $P\left(Z_{p-q}^{11} \in \mathcal{I}_{p-q} \mid C_{i}^{1}\right)$ affects $P\left(C_{i}^{1} \mid Z_{p-q}^{11} \in \mathcal{I}_{p-q}\right)$. In fact, $P\left(C_{i}^{1} \mid Z_{p-q}^{11} \in \mathcal{I}_{p-q}\right)$ is obtained using the Bayesian equation in (2) and $P\left(Z_{p-q}^{11} \in \mathcal{I}_{p-q} \mid C_{i}^{1}\right)$ from (11) by assuming $C_{i}^{1}$ is uniform across each cell as prior knowledge.

For $P\left(Z_{p-q}^{10} \in \mathcal{I}_{p-q} \mid C_{i}^{1}\right)$, we have the following result.

Lemma 3:

$$
\begin{aligned}
& P\left(Z_{p-q}^{10} \in \mathcal{I}_{p-q} \mid C_{i}^{1}\right)= \\
& \frac{1}{\eta^{10}}\left(1-\int_{\tilde{z}_{p}-\tilde{z}_{q}}^{\tilde{z}_{p}-\tilde{z}_{q}+1} \mathrm{~F}_{Z_{p-q}^{11} \mid C_{i}^{1}}(z) d z\right)
\end{aligned}
$$

where $\eta^{10}$ is the normalizing factor.

The proof of Lemma 3 is in Appendix B. This result also does not depend on source transmission power. Using the similar process that computes the results shown in Fig. 3(a), Fig. 3(b) illustrates the corresponding posterior probability $P\left(C_{i}^{1} \mid Z_{p-q}^{10} \in \mathcal{I}_{p-q}\right)$ for $P\left(Z_{p-q}^{10} \in \mathcal{I}_{p-q} \mid C_{i}^{1}\right)$.

At last, we compute $P\left(Z_{p-q}^{00} \in \mathcal{I}_{p-q} \mid C_{i}^{1}\right)$, and we have the following lemma:

Lemma 4:

$$
P\left(Z_{p-q}^{00} \in \mathcal{I}_{p-q} \mid C_{i}^{1}\right)=\frac{1}{\eta^{00}}
$$

Proof: The proof is similar to that of Lemma 2. Note that neither robots have reception. According to (4), we have

$$
\mu_{p}-\mu_{q}=\zeta-\zeta=0
$$

and the PDF of $Z_{p-q}^{00} \mid C_{i}^{1}$ is

$$
f_{Z_{p-q}^{00} \mid C_{i}^{1}}\left(z_{p-q}^{00}\right)=\operatorname{Bel}\left(0,2 \sigma^{2}\right) .
$$

Since the PDF $f_{Z_{p-q}^{00} \mid C_{i}^{1}}\left(z_{p-q}^{00}\right)$ is not a function of the distance to cell $i$, we have

$$
\frac{1}{\eta^{00}}=\int_{\tilde{z}_{p}-\tilde{z}_{q}-1}^{\tilde{z}_{p}-\tilde{z}_{q}+1} f_{Z_{p-q}^{00} \mid C_{i}^{1}}(z) d z .
$$

Intuitively, $P\left(Z_{p-q}^{00} \in \mathcal{I}_{p-q} \mid C_{i}^{1}\right)$ cannot provide more information regarding the whereabouts of $i$, except that it is located outside the sensing range. Therefore, the probability cannot be a function of the distance to the active cell $i$.

\section{Sensor Fusion of Multiple Pairs}

Now, we are ready to show that the $m$-dimensional joint conditional probability $P\left(\tilde{\mathbf{Z}}^{k}=\tilde{\mathbf{z}}^{k} \mid C_{i}^{1}\right)$ can be reduced to a combination of pairwise conditional probabilities $P\left(Z_{p} \in \mathcal{I}_{p}, Z_{q} \in\right.$ $\left.\mathcal{I}_{q} \mid C_{i}^{1}\right)$. We have the following lemma:

Lemma 5:

$$
P\left(\tilde{\mathbf{Z}}=\tilde{\mathbf{z}} \mid C_{i}^{1}\right)=\frac{1}{\eta} \prod_{(p, q) \in \mathcal{E}} P\left(Z_{p} \in \mathcal{I}_{p}, Z_{q} \in \mathcal{I}_{q} \mid C_{i}^{1}\right)
$$

where $\eta$ is the normalizing factor and remains the same for all $p$ and $q$ values.

Proof:

$$
P\left(\tilde{\mathbf{Z}}=\tilde{\mathbf{z}} \mid C_{i}^{1}\right)=\prod_{l=1}^{m} P\left(\tilde{Z}_{l}=\tilde{z}_{l} \mid C_{i}^{1}\right) .
$$

The individual conditional probability $P\left(\tilde{Z}_{l}=\tilde{z}_{l} \mid C_{i}^{1}\right)$ can be paired up as

$$
\begin{aligned}
P\left(\tilde{\mathbf{Z}}=\tilde{\mathbf{z}} \mid C_{i}^{1}\right)= & \prod_{l=1}^{m} P\left(Z_{l} \in \mathcal{I}_{l} \mid C_{i}^{1}\right) \\
= & \prod_{l=1}^{m}\left(\frac{P\left(Z_{l} \in \mathcal{I}_{l} \mid C_{i}^{1}\right)^{(m-1)}}{P\left(Z_{l} \in \mathcal{I}_{l} \mid C_{i}^{1}\right)^{(m-2)}}\right) \\
= & \frac{1}{\prod_{l=1}^{m} P\left(Z_{l} \in \mathcal{I}_{l} \mid C_{i}^{1}\right)^{(m-2)}} \\
& \times \prod_{p=1}^{m-1} \prod_{q=p+1}^{m} P\left(Z_{p} \in \mathcal{I}_{p} \mid C_{i}^{1}\right) \\
& \times P\left(Z_{q} \in \mathcal{I}_{q} \mid C_{i}^{1}\right) \\
= & \frac{1}{\eta} \prod_{(p, q) \in \mathcal{E}} P\left(Z_{p} \in \mathcal{I}_{p}, Z_{q} \in \mathcal{I}_{q} \mid C_{i}^{1}\right)
\end{aligned}
$$

is a nonzero constant. 


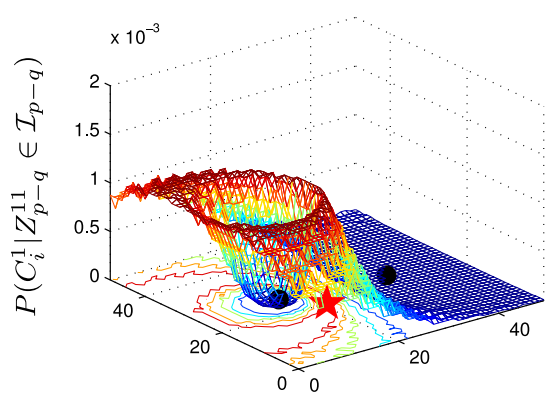

(a)

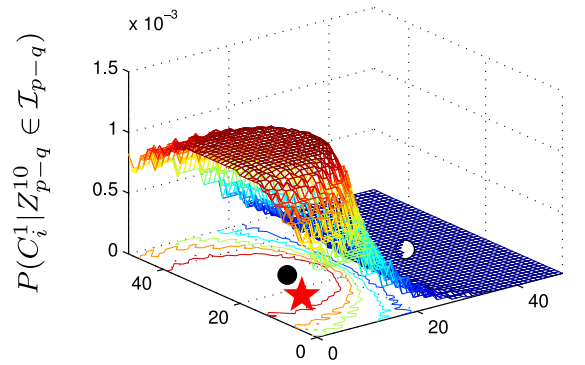

(b)

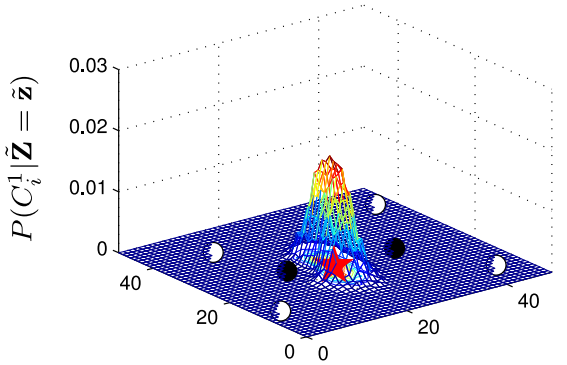

(c)

Fig. 3. Sample cases of posterior condition distributions of the signal source location given that $P\left(C_{i}^{1}\right)$ initially uniform across cells. (a) Dual detection, (b) single detection, and (c) fusion of all pairs. The red star is the active radio source location. These illustrations are obtained using $P\left(Z_{p-q} \in \mathcal{I}_{p-q} \mid C_{i}^{1}\right)$ and the Bayesian framework in (2). The grid size is $50 \times 50$. Black and white dots represent robots with and without receptions, respectively. Note that the posterior probability in (a) looks like a volcanic crater while that of (b) looks like a plateau.

where $\eta=\prod_{l=1}^{m} P\left(Z_{l} \in \mathcal{I}_{l} \mid C_{i}^{1}\right)^{(m-2)}$ remains the same for all $p$ and $q$ values.

Now, we are ready to complete the sensing model $P(\tilde{\mathbf{Z}}=$ $\left.\tilde{\mathbf{z}} \mid C_{i}^{1}\right)$ by combining results in Lemmas $1-5$, we have the following theorem:

Theorem 1: The high-dimensional joint conditional probability sensing model $P\left(\tilde{\mathbf{Z}}=\tilde{\mathbf{z}} \mid C_{i}^{1}\right)$ can be decomposed as a combination of pairwise conditional probabilities

$$
\begin{aligned}
P\left(\tilde{\mathbf{Z}}=\tilde{\mathbf{z}} \mid C_{i}^{1}\right)= & \frac{1}{\eta^{\prime \prime}} \prod_{(p, q) \in \mathcal{E}_{11}}\left(\begin{array}{c}
\mathrm{F}_{Z_{p-q}^{11} \mid C_{i}^{1}}\left(\tilde{z}_{p}-\tilde{z}_{q}+1\right) \\
-\mathrm{F}_{Z_{p-q}^{11} \mid C_{i}^{1}}\left(\tilde{z}_{p}-\tilde{z}_{q}-1\right)
\end{array}\right) \\
& \prod_{(p, q) \in \mathcal{E}_{10}}\left(1-\int_{\tilde{z}_{p}-\tilde{z}_{q}}^{\tilde{z}_{p}-\tilde{z}_{q}+1} \mathrm{~F}_{Z_{p-q}^{11} \mid C_{i}^{1}}(z) d z\right)
\end{aligned}
$$

where $\eta^{\prime \prime}$ is the normalizing factor and remains the same for all $p$ and $q$ values.

Proof: Combining Lemma 1 with Lemma 5, the sensing model becomes

$$
\begin{aligned}
P(\tilde{\mathbf{Z}} & \left.=\tilde{\mathbf{z}} \mid C_{i}^{1}\right)=\frac{1}{\eta} \prod_{(p, q) \in \mathcal{E}} \frac{1}{\eta_{p q}} P\left(Z_{p-q} \in \mathcal{I}_{p-q} \mid C_{i}^{1}\right) \\
& =\left(\frac{1}{\eta} \prod_{(p, q) \in \mathcal{E}} \frac{1}{\eta_{p q}}\right) \prod_{(p, q) \in \mathcal{E}} P\left(Z_{p-q} \in \mathcal{I}_{p-q} \mid C_{i}^{1}\right) \\
& =\frac{1}{\eta^{\prime}} \prod_{(p, q) \in \mathcal{E}} P\left(Z_{p-q} \in \mathcal{I}_{p-q} \mid C_{i}^{1}\right)
\end{aligned}
$$

where $\eta^{\prime}=\eta \prod_{(p, q) \in \mathcal{E}} \eta_{p q}$ is the normalizing factor and remains the same for all $p$ and $q$ values.

Applying (8) to (20) and combining Lemmas 2 and 3, the sensing model is rewritten as

$$
\begin{aligned}
P\left(\tilde{\mathbf{Z}}=\tilde{\mathbf{z}} \mid C_{i}^{1}\right)= & \frac{1}{\eta^{\prime}} \prod_{(p, q) \in \mathcal{E}_{11}} P\left(Z_{p-q}^{11} \in \mathcal{I}_{p-q} \mid C_{i}^{1}\right) \\
& \times \prod_{(p, q) \in \mathcal{E}_{10}} P\left(Z_{p-q}^{10} \in \mathcal{I}_{p-q} \mid C_{i}^{1}\right)
\end{aligned}
$$

$$
\begin{aligned}
& \times \prod_{(p, q) \in \mathcal{E}_{00}} P\left(Z_{p-q}^{00} \in \mathcal{I}_{p-q} \mid C_{i}^{1}\right) \\
= & \frac{1}{\eta^{\prime \prime}} \prod_{(p, q) \in \mathcal{E}_{11}}\left(\begin{array}{c}
\mathrm{F}_{Z_{p-q}^{11} \mid C_{i}^{1}}\left(\tilde{z}_{p}-\tilde{z}_{q}+1\right) \\
-\mathrm{F}_{Z_{p-q}^{11} \mid C_{i}^{1}}\left(\tilde{z}_{p}-\tilde{z}_{q}-1\right)
\end{array}\right) \\
& \times \prod_{(p, q) \in \mathcal{E}_{10}} \\
& \times\left(1-\int_{\tilde{z}_{p}-\tilde{z}_{q}}^{\tilde{z}_{p}-\tilde{z}_{q}+1} \mathrm{~F}_{Z_{p-q}^{11} \mid C_{i}^{1}}(z) d z\right)
\end{aligned}
$$

where

$$
\begin{aligned}
\eta^{\prime \prime} & =\frac{\eta^{\prime} \prod_{(p, q) \in \mathcal{E}_{10}} \eta^{10}}{\prod_{(p, q) \in \mathcal{E}_{00}} P\left(Z_{p-q}^{00} \in \mathcal{I}_{p-q} \mid C_{i}^{1}\right)} \\
& =\eta^{\prime}\left(\prod_{(p, q) \in \mathcal{E}_{10}} \eta^{10}\right)\left(\prod_{(p, q) \in \mathcal{E}_{00}} \eta^{00}\right)
\end{aligned}
$$

is the normalizing factor.

Again, Fig. 3(c) illustrates the posterior probability $P\left(C_{i}^{1} \mid \tilde{\mathbf{Z}}=\tilde{\mathbf{z}}\right)$ for the six-robot case. The corresponding $P(\tilde{\mathbf{Z}}=$ $\left.\tilde{\mathbf{z}} \mid C_{i}^{1}\right)$ is computed from the fusion of all pairs with RSS readings. Note that only two of the six has RSS readings due to their range limit. Therefore, there is one pair in $\mathcal{E}_{11}$ and eight pairs in $\mathcal{E}_{10}$. As we can see, the resulting $P\left(C_{i}^{1} \mid \tilde{\mathbf{Z}}=\tilde{\mathbf{z}}\right)$ is a unimodal spatial distribution with the peak close to the actual signal source, which is desirable.

\section{RoBot Motion PlanNer}

\section{A. Path Planning for Each Robot Pair}

Theorem 1 summarizes how to compute $P\left(\tilde{\mathbf{Z}}=\tilde{\mathbf{z}} \mid C_{i}^{1}\right)$. With the sensing model, the Bayesian framework in (2) can derive the posterior source location distributions $P\left(C_{i} \mid \tilde{\mathbf{Z}}=\tilde{\mathbf{z}}\right)$. This completes the sensing part in Fig. 2. The next step is to develop a multirobot motion planner that enables robots to quickly localize radio sources using the SPOG. We build on the ridge walking algorithm (RWA) in [30]. RWA has been designed for 


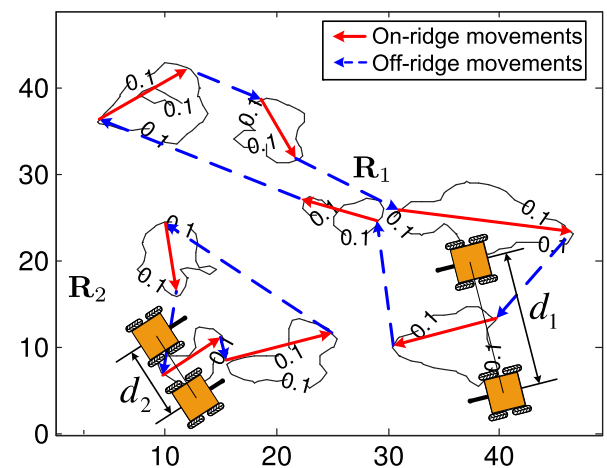

Fig. 4. Level sets with probability threshold of 0.1 , ridges, and PRWA with two pairs of robots over a $50 \times 50$ grid.

a single robot without sensing range limit to localize multiple radio sources. The experimental results have shown that it is an efficient framework. However, RWA is not designed for multiple robots and significant revisions are needed. Let us begin with a brief review of RWA.

RWA uses a probability threshold plane that intercepts $P\left(C_{i} \mid \tilde{\mathbf{Z}}=\tilde{\mathbf{z}}\right)$ to generate level sets that enclose all cells with $P\left(C_{i} \mid \tilde{\mathbf{Z}}=\tilde{\mathbf{z}}\right)$ no less than the threshold $p, p \in(0,1]$. Level set $S(p)$ is introduced as follows:

$$
S(p)=\left\{i \mid P\left(C_{i} \mid \tilde{\mathbf{Z}}_{j}^{k}=\tilde{\mathbf{z}}_{j}^{k}\right) \geq p, i \in \mathcal{N}\right\} .
$$

$S(p)$ contains all cells with $P\left(C_{i} \mid \tilde{\mathbf{Z}}_{j}^{k}=\tilde{\mathbf{z}}_{j}^{k}\right)$ above the probability threshold plane. $S(p)$ usually consists of several disconnected components. Define $s_{\max }$ as the total number of the disconnected components and $S_{s}$ as the $s$ th component, $s=1, \ldots, s_{\max }$. Therefore, $S(p)=\cup_{s=1}^{s_{\max }} S_{s}$ and $S_{s} \cap S_{h}=\emptyset$, where $h \neq s$ and $h=1,2, \ldots, s_{\max }$. For the $s$ th component, we define its ridge $R_{s}$ as the line segment defined by points $\mathbf{x}^{\prime}=\left[\mathbf{x}^{\prime}, \mathbf{y}^{\prime}\right]^{\mathbf{T}}$ and $\mathbf{x}^{\prime \prime}=\left[\mathbf{x}^{\prime \prime}, \mathbf{y}^{\prime \prime}\right]^{\mathbf{T}}$ on $S_{s}$

$$
\begin{aligned}
R_{s} & =\left\{[x, y]^{T} \mid x=(1-\alpha) x^{\prime}+\alpha x^{\prime \prime}\right. \\
y & \left.=(1-\alpha) y^{\prime}+\alpha y^{\prime \prime}, \alpha \in[0,1]\right\}
\end{aligned}
$$

where points $\mathrm{x}^{\prime}$ and $\mathrm{x}^{\prime \prime}$ are the two points on $S_{s}$ such that the distance between $\mathrm{x}^{\prime}$ and $\mathrm{x}^{\prime \prime}$ is the maximum.

The irregular closed curves in Fig. 4 are examples of level sets. Ridges are created by extracting the longest dimension of each isolated level set. The directed solid red line segments in Fig. 4 are ridges. In RWA, a three-opt heuristics algorithm is employed to compute an Euclidean TSP tour for the single robot that must include all ridges. The TSP tour is partitioned into on-ridge and off-ridge segments. For off-ridge segments, the robot moves at its fastest speed. For on-ridge segments, the robot spends the time proportional to the summation of posterior conditional probability $P\left(C_{i} \mid \tilde{\mathbf{Z}}=\tilde{\mathbf{z}}\right)$ over the corresponding isolated level set on each ridge. This means that the robot spends more time in high-probability regions, which increases the localization efficiency.

Since we have more than one robot, we need many subtours instead of a single TSP tour. We pair up robots and treat a pair of robots as a super robot. Recall that $m$ is an even number, there are $m / 2$ super robots. Therefore, we need to partition the TSP tour into $m / 2$ subtours and assign each super robot to a subtour. The partition is based on the $k$-means clustering algorithm [37] with $m / 2$ as the cluster number to cluster ridge sets. For each cluster, we again use a three-opt heuristics algorithm to find the TSP and the rest of RWA follows. Hence, we call this approach the pairwise ridge walking algorithm (PRWA).

It is worth noting that the reason for using the three-opt heuristics algorithm is to facilitate the derivation of the worst case computation complexity result. In fact, there are many solvers, such as Concorde TSP Solver [38], that are faster than the threeopt algorithm in practice. The framework can be easily extended to accommodate those solvers.

\section{B. Determine Intrapair Distance}

The remaining issue is how to determine the distance between each paired robots. Comparing Fig. 3(a) and (b), we notice that the dual detection case provides more information (less uncertainty) about radio source locations than the single detection case does. Determining the optimal intrapair is done by minimizing the uncertainty of radio source locations. The Shannon entropy over a spatial distribution of radio source locations can be used to measure the location uncertainty. High entropy value means high uncertainty. For example, radio sources are assumed to be evenly distributed across the entire searching region at the initial step which corresponds to the highest entropy value. As indicated by the decreasing entropy values, the spatial distribution of radio sources gradually peaks at vicinities of radio sources as more readings are received. Any decisions that lead to a small entropy function value provide more accurate localization results. Therefore, we choose distance $d_{u}^{*}$ between the $u$ th pair by minimizing the Shannon entropy.

Define $\mathcal{S}_{u}$ as the set of cells in the isolated level set that correspond to the ridge cluster $\mathbf{R}_{u}$. Let cell $v \in \mathcal{S}_{u}$. Assume that the radio source $\mathbf{x}_{v}=\left[x_{v}, y_{v}\right]^{T}$ is located at the center of cell $C_{v}$ by ignoring the minor intracell difference. By defining $\hat{z}_{l v}^{w}$ as the mean RSS reading at robot $l$, we have

$$
\hat{z}_{l v}^{w}=w-10 \beta \log _{10}\left(d_{l v}\right)
$$

where $w \in\left[w_{\min }, w_{\max }\right]$ is the unknown source transmission power, which varies from $w_{\min }$ to $w_{\max }$.

Define $\hat{\mathbf{Z}}_{v}^{w}=\left[\hat{Z}_{p v}^{w}, \hat{Z}_{q v}^{w}\right]^{T}$ as the RSS readings for the robot pair. Define $r_{u}(t)$ as the center position of the robot pair at time $t$. We know $r_{u}(t)$ because PRWA provides the trajectory for the super robot using the center position of the robot pair as the position on the trajectory. Denote $P\left(C_{i} \mid \hat{\mathbf{Z}}_{v}^{w}=\hat{\mathbf{z}}_{v}^{w}, r_{u}(t), d_{u}\right)$ as the posterior probability that cell $i$ contains a radio source given $\hat{\mathbf{z}}_{v}^{w}, \mathbf{r}_{\mathbf{u}}(\mathbf{t})$, and $d_{u}$. Define $H\left(t, w, v, d_{u}\right)$ as the Shannon entropy over the probability distribution $P\left(C_{i} \mid \hat{\mathbf{Z}}_{v}^{w}=\hat{\mathbf{z}}_{v}^{w}, r_{u}(t), d_{u}\right)$, given $v, w$, and $d_{u} . H\left(t, w, v, d_{u}\right)$ is given by

$$
H\left(t, w, v, d_{u}\right)=-\sum_{i \in \mathcal{S}_{u}}\left(\begin{array}{c}
P\left(C_{i} \mid \hat{\mathbf{Z}}_{v}^{w}=\hat{\mathbf{z}}_{v}^{w}, r_{u}(t), d_{u}\right) \\
\times \ln P\left(C_{i} \mid \hat{\mathbf{Z}}_{v}^{w}=\hat{\mathbf{z}}_{v}^{w}, \mathbf{r}_{\mathbf{u}}(\mathbf{t}), \mathbf{d}_{\mathbf{u}}\right)
\end{array}\right)
$$




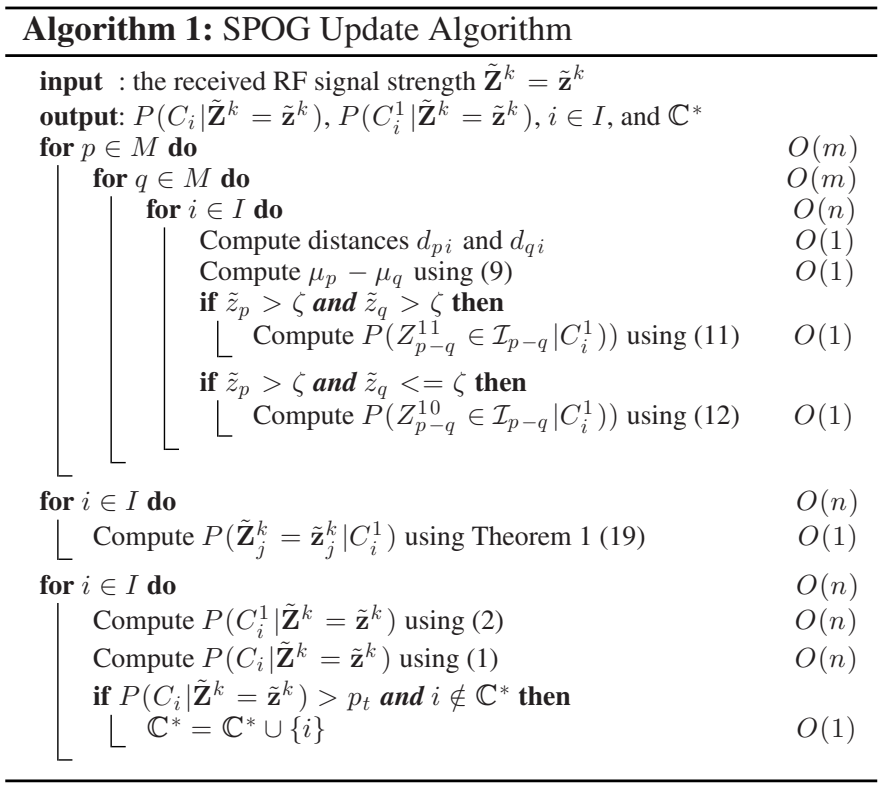

where $P\left(C_{i} \mid \hat{\mathbf{Z}}_{v}^{w}=\hat{\mathbf{z}}_{v}^{w}, r_{u}(t), d_{u}\right)$ is obtained from (1) and (2) after calculating the sensing model (19) with $\hat{\mathbf{z}}_{v}^{w}$. We choose the optimal $d_{u}^{*}$ that minimizes the following Shannon entropy for the cluster region over the period $\tau_{u}$ when the robot is inside $R_{u}$ :

$$
d_{u}^{*}=\arg \min _{d_{u}} \int_{t}^{t+\tau_{u}} \sum_{w=w_{\min }}^{w_{\max }} \sum_{v \in \mathcal{S}_{u}} H\left(t, w, v, d_{u}\right) .
$$

Note that here, we assume that $w$ is evenly distributed over integer values in $\left[w_{\min }, w_{\max }\right]$. In fact, we can estimate the more accurate distribution of $w$ once more received signals become available to improve the model.

Remark 2: It is worth noting that the partition of $m$ robots into $m / 2$ in the motion planning part does not change it in sensing part, where we still need to examine all $\left(\begin{array}{c}m \\ 2\end{array}\right)$ pairs to find pairs with RSS readings to perform sensor fusion. This is also shown later in Algorithm 1.

\section{AlgorithmS}

To summarize our analysis, we present two algorithms, including an SPOG update algorithm and the PRWA. Corresponding to the sensing problem in Section IV, the SPOG update algorithm runs when a radio signal is detected. Define set $\mathbb{C}^{*}$ as the set of cells that contain radio sources with initial value $\mathbb{C}^{*}=\emptyset$. Define $p_{t}$ as the probability threshold for finding the radio source. The robot reports the cells that satisfy $P\left(C_{i} \mid \tilde{\mathbf{Z}}^{k}=\tilde{\mathbf{z}}^{k}\right)>p_{t}$ as the cells that contain at least one radio source.

Recall that $n$ is the total number of cells, and $m$ is the total number of robots. It is clear that the SPOG update algorithm runs $O\left(n m^{2}+n^{2}\right)$. The initial value settings are $P\left(C_{i} \mid \tilde{\mathbf{Z}}_{0}^{0}=\right.$ $\left.\tilde{\mathbf{z}}_{0}^{0}\right)=0$ and $P\left(C_{i}^{1} \mid \tilde{\mathbf{Z}}_{0}^{0}=\tilde{\mathbf{z}}_{0}^{0}\right)=1 / n$.

The PRWA algorithm runs every $\tau_{0}$ time. Define $D=$ $\left\{1, \ldots, d_{\max }\right\}$ as the index set of the distance between pairwise robots, where $d_{\max }$ is the distance resolution. Let $t_{\max }=$ $\frac{t^{k+1}-t^{k}}{\Delta t}$ be the time resolution, where $\Delta t$ is the time step. Define

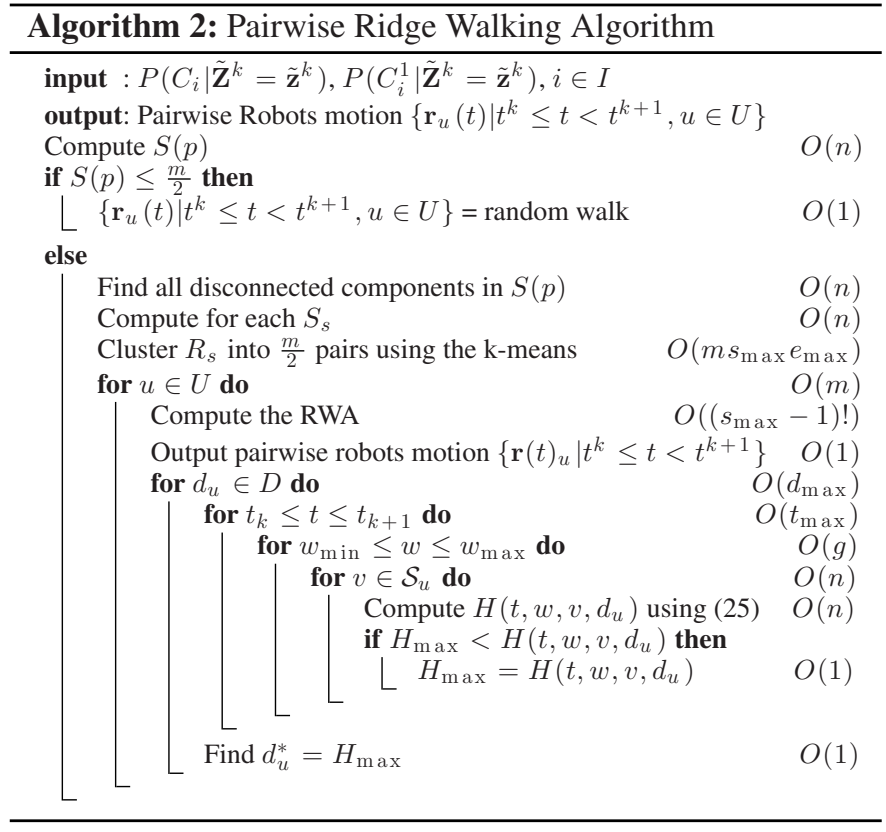

$g=w_{\max }-w_{\min }$ as the total number of transmission power level.

As illustrated in Algorithm 2, the pairwise robots perform a random walk until set $S(p) \geq \frac{m}{2}$ at the initialization stage. Then, the pairwise robots switch into the normal ridge walking mode. The robots stop when no additional radio source has been found in $k_{\max }$ consecutive periods, where $k_{\max }$ is a preset iteration number. Algorithm uses the $k$-means clustering algorithm to partition ridges into robot pairs and its complexity is $O\left(m s_{\max } e_{\max }\right)$, where $e_{\max }$ is the maximum number of iteration. The overall complexity is $O\left(m d_{\max } t_{\max } g n^{2}+\right.$ $\left.m s_{\max } e_{\max }+m\left(s_{\max }-1\right) !\right)$. Since $s_{\max }$ refers to the maximum number of disconnected components in the $S(p)$ and usually is a small number, the speed of this algorithm is not a concern.

A natural concern is how the two algorithms scale against the number of robots, $m$, and the number of cells, $n$. From the analysis, we know that the PRWA is linear to $m$, and the SPOG update algorithm is quadratic in $m$. Both algorithms are quadratic in $n$. One would concern an overall complexity of $O\left(n m^{2}+n^{2}\right)$ could be expensive for the SPOG update algorithm. Let us show why such a case is not a significant concern:

There are two ways to increase $n: 1)$ finer grid with smaller cells for the same searching region or 2) larger searching region using the same cell size. For 1), there is a lower bound of how small each grid cells can be due to the limited resolution of the antenna. Therefore, $n$ cannot be arbitrarily large for this case. For 2), this generates a sparse robot distribution for the same number of robots. For the case, many pairs are actually the 00 type according to (8), which are handled by constant terms in the sensing model according to Theorem 1 and, hence, do not require explicit computation. Therefore, the actual complexity regarding $m$ is somewhere between the linear case and the quadratic case. The sparser the robot distribution gets, the SPOG algorithm behaves closer to the linear case. Therefore, 
the overall complexity is far less than $O\left(n m^{2}+n^{2}\right)$. In either case, we also cannot arbitrarily increase $m$ for a given searching region because the number of robots needed is always less than the ratio between overall searching area and antenna coverage area. If radio sources are not evenly distributed across the searching region, then the ratio is even smaller because the overall searching area should be replaced by adjacent areas occupied by radio sources. Therefore, there is a natural upper bound for $m$. If such bound is reached, the robot team does not need to move because the team already provides full coverage of the entire field or all adjacent regions of radio sources. In fact, more important and realistic cases are when resource is constrained by a small number of robots.

\section{EXPERIMENTS}

To validate the algorithms, we have conducted both simulation and physical experiments. We have implemented the algorithms and the simulation platform using Microsoft Visual C++ .NET 2005 with OpenGL on a PC Desktop. The radio sources are XBee Pro with ZigBeeT/802.15.4 OEM radio frequency modules [see Fig. 6(c)] produced by Digi International Inc. The antenna is calibrated first with the radio sources. The calibration establishes the parameters in (3).

\section{A. Simulation}

We use the data from the real hardware to drive the simulation experiments below.

The grid is a square with $50 \times 50$ cells. Each grid cell has a size of $50.0 \times 50.0 \mathrm{~cm}^{2}$. Each radio source generates radio transmission signals according to an i.i.d. Poisson process with a rate of $\lambda=0.05$ packets per second. We choose the probability convergence threshold as $p_{t}=0.9$ which means if $P\left(C_{i} \mid \tilde{\mathbf{Z}}=\tilde{\mathbf{z}}\right)>0.9$, the algorithm outputs the cell as a radio source location. During each trial of the simulation, we randomly generate radio source locations in the grid and randomly set their power levels as one of five power levels offered by XBee Pro nodes.

We compare the PRWA algorithm with four heuristics. Two of the four heuristics are based on random walk: a pairwise random walk and a regular random walk. In the pairwise random walk, robots are paired just as PRWA does. Each pair is treated as a super robot to perform a random walk together while all robots perform independent movements in the regular random walk. The remaining two heuristics are based on a fixed-route patrol: The robots patrol the field using a predefined route that covers the search region. Again, robots are either paired, which results in a pairwise patrol, or nonpaired, which results in a regular patrol. Robot pairs in the pairwise patrol or individual robots in the regular patrol are distributed evenly along the route to increase coverage.

The experiment compares all five methods under different numbers of radio sources and robots. Fig. 5(a) and (b) illustrates experiment results. Each data point is an average of 100 independent trials. The results show that PRWA is consistently the fastest method under all comparisons. This is reflected by its small average localization times and small standard deviations.

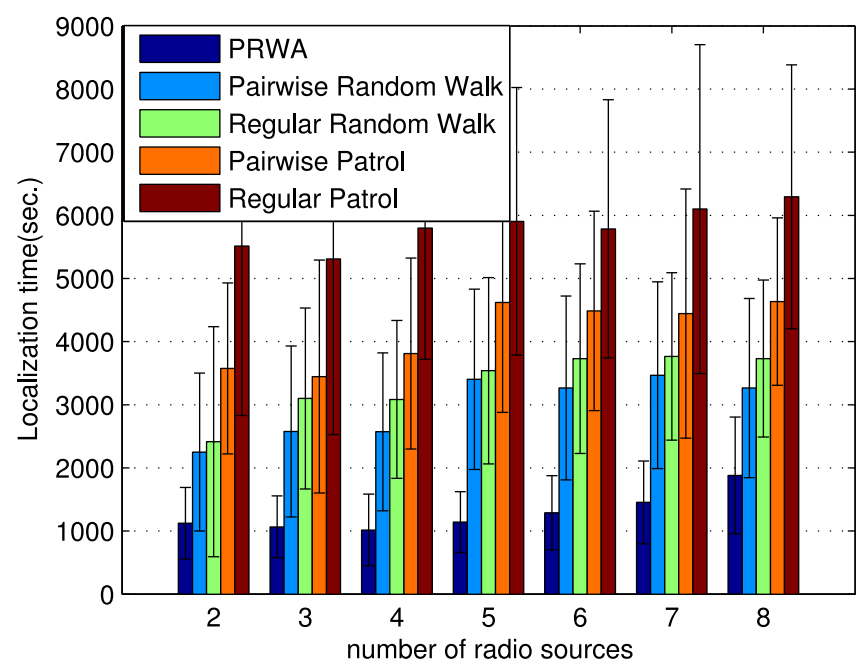

(a)

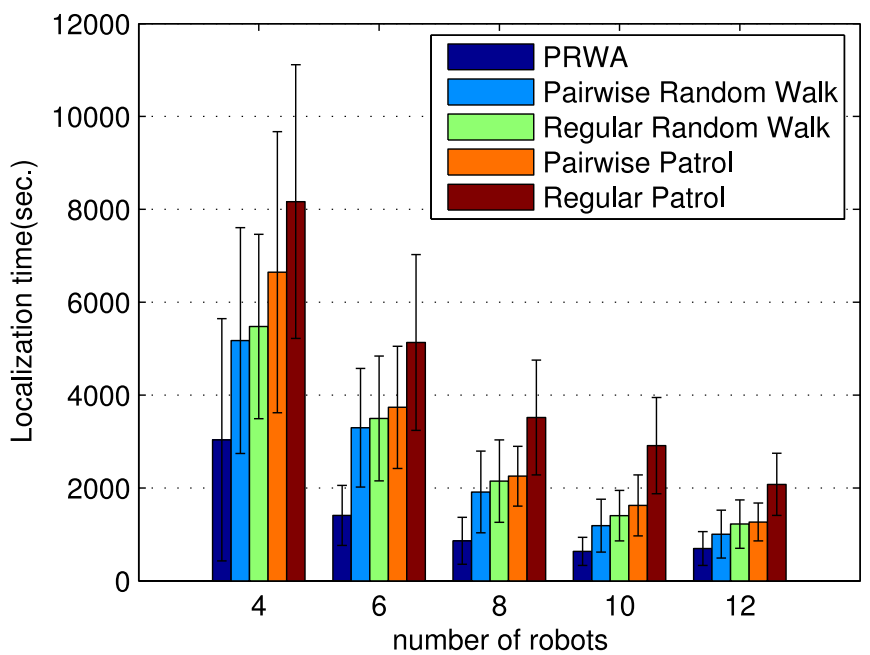

(b)

Fig. 5. Simulation results: (a) Localization time versus number of radio sources. The number of robots used is 6. (b) Localization time versus number of robots. There are four radio sources. Both average localization times are in colored bars, and their standard deviations in vertical line segments are illustrated here.

In addition, the pairwise random walk and the pairwise patrol are consistently faster than the regular random walk and patrol, respectively. This is expected because paired robots are more efficient with their limited sensing ranges. Another interesting observation is that the two random walk-based methods are faster than the two fixed-route patrol methods. This is expected because random walk can bring robots together from time to time, which increases the number of effective pairs and hence listening efficiency. The fixed-route patrol methods emphasize coverage and spread robot pairs or individual robots apart along the route and, hence, cannot create many effective pairs, which decreases localization efficiency. The results in Fig. 5(b) also show that the difference between the five methods decreases as the number of robots increases. However, in reality, the number of robots is often constrained to where PRWA is superior. 


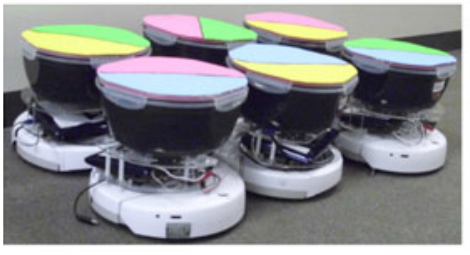

(a)

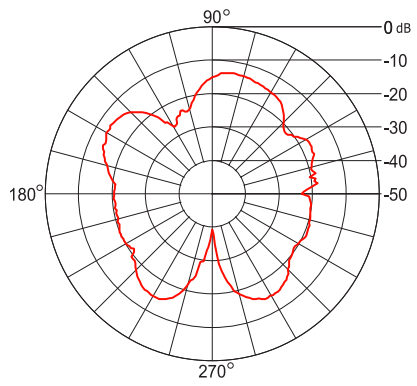

(c)

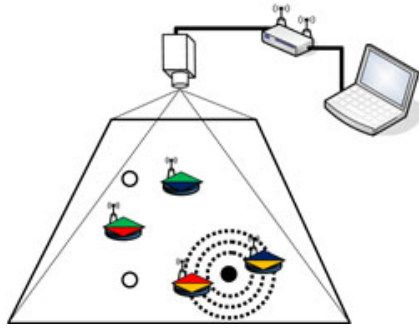

(d)
Fig. 6. Hardware and system setup for the physical experiments. (a) Robot team consists of six modified iRobot Create robots. (b) Sample radio source. (c) Radiation pattern of the chip antenna of the radio source. (d) System setup during the experiment.

\section{B. Physical Experiments}

In the physical experiment, we have modified six iRobot Create robots [see Fig. 6(a)]. The color patches on the top of the robots are used for robot identification in video. Each robot measures $34.0 \mathrm{~cm}$ in diameter and $30.0 \mathrm{~cm}$ in height. The maximum speed of the robot is $40.0 \mathrm{~cm} / \mathrm{s}$. The maximum battery life is about $2 \mathrm{~h}$. Only four robots are used in the actual experiments with the remaining two as backup robots to deal with the hardware reliability issue in the long experiment.

The radio sources are XBee Pro with ZigBeeT/802.15.4 OEM radio frequency modules [see Fig. 6(b)] with on-board chip antennas. The radiation pattern of the chip antennas is illustrated in Fig. 6(c). According to the antenna theory, the radiation pattern of an antenna is also its reception pattern. Both the robot and the radio source use the same radio module with the same built-in chip antennas. In addition, we have strong interference from WiFi signals from the nearby building. We have to tune down the gain for the Xbee antenna to overcome the interference. This is equivalent to raising the background noise threshold $\zeta$ in (4). It turns out that the effective listening radius is less than $1 \mathrm{~m}$. To overcome the issue that the radiation pattern is not perfectly round, we have calibrated Xbee antenna and fit a circle to obtain an approximation represented by mean radius for the chip antenna. The variations between the actual radiation level and the mean reception calculated from the mean radius are used to compute the variance of noise, $\sigma$, which captures both radiation pattern deviation and other noises.

The test field is a square with a side length of $10.00 \mathrm{~m}$, which has been divided into $50 \times 50$ equal-sized square cells. In the test, we have three unknown radio sources transmitting at 0.1 packets per second each. The source transmission power can

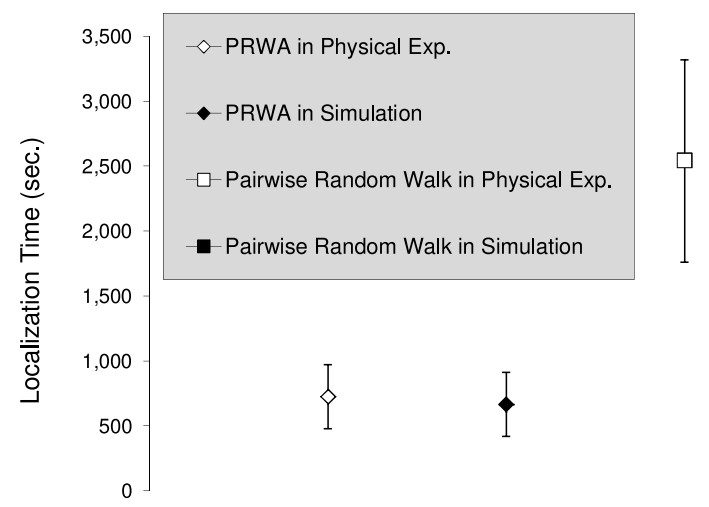

Fig. 7. Physical experiment results. The marker position is the average value. The vertical bars correspond to $[-\sigma, \sigma]$ with $\sigma$ as the standard deviation.

vary at different levels. As shown in Fig. 6(d), an overhead camera has been used to provide the robots with position information at $16 \mathrm{~Hz}$ using motion detection methods. The camera is mounted at the third floor of a nearby building. The camera is an Arecont Vision 3100 networked video camera. The location accuracy is within $\pm 10.0 \mathrm{~cm}$.

Due to the battery life limit, we cannot compare PRWA with all four heuristics in the physical experiments. We only compare PRWA with the pairwise random walk. The localization time of pairwise random walk appears to be second only to the PRWA in simulation and, hence, does not demand too much in battery life. In the experiment, the probability threshold $p_{t}$ is set to be 0.9 . The results of physical experiments in comparison with simulation are shown in Fig. 7. It is worth noting that the simulation is carried out using the same parameter settings, the same calibrated antenna models, and the same field setup to make the simulation as faithful to the physical experiments as possible. Due to constraints on resources and time, we can only conduct physical experiments for five trials for each data point in Fig. 7. Actually, each trial takes at least 3-4 h if including set up time. Even from the limited results, we can see that physical experiment data are very close to that of simulation results. This is anticipated because the simulation is driven by the data from the same hardware. It is consistent that both physical experiments and simulation results agree that PRWA is faster than the pairwise random walk in localization speed. In addition, the standard deviation of PRWA localization time is much smaller than that of the pairwise random walk. This means that PRWA is both faster and more predictable.

To further illustrate the results, we have attached a video clip showing a sample trial of the physical experiment. The robot trajectories are visualized using the real robot movement data and probability values are shown in grayscale. As shown in the results, the on-ridge movements appear near radio sources. At the end of the experiments, the estimated locations of radio sources are within the error range of the actual locations. The localization process is successful. It is also worth noting that the probability map has noisy peaks caused by environment reflections. 


\section{CONCLUSION AND Future WORK}

We have reported a new localization method that enables a team of mobile robots to localize multiple unknown transient radio sources. To cope with the challenges from signal correspondence, limited sensing ranges, and unknown transmission power, we paired up robots and developed a sensing model using RSS ratios from robot pairs. Since a conventional continuous tracking algorithm cannot be applied due to signal intermittency and anonymity, we employed a Bayesian-type approach by extending the existing SPOG framework to multiple robot pairs. To handle the consequent issue that high-order belief space is difficult to compute, we formally proved that the joint conditional posterior probability of source locations for the $m$-robot team can be obtained by combining pairwise joint conditional posterior probabilities. Moreover, we proposed a PRWA to coordinate the robot pairs based on the clustering of high-probability regions and the minimization of local Shannon entropy. We implemented the algorithm and tested it under the hardware-driven simulation and physical experiments. Results show that PRWA-based localization consistently outperforms the other four heuristics in all settings tested.

In the future, we will address the decentralized control issue by proving that the joint posterior probability updating process can be handled locally in the distributed pairs. We will study how the information exchange rate between pairs affects convergence speed to provide theoretical bounds on the search time of the distributed approaches. In addition, robot pairs can be viewed as a rudimentary way of teaming. We will also explore other teaming topology and size for the shortest search time.

\section{APPENDIX A}

\section{PROOF OF LEMMA 1}

Recall that $Z_{p-q}=Z_{p}-Z_{q}$, and define $Z_{p+q}:=Z_{p}+Z_{q}$. Denote $\mathbf{A}=\left[\begin{array}{cc}1 & -1 \\ 1 & 1\end{array}\right]$ as the linear transformation matrix. Therefore

$$
\left[\begin{array}{c}
Z_{p-q} \\
Z_{p+q}
\end{array}\right]=\mathbf{A}\left[\begin{array}{c}
Z_{p} \\
Z_{q}
\end{array}\right] .
$$

Let us define $R_{A}$ as the transformed integral region. According to [39], matrix $\mathbf{A}$ transforms the joint PDF of random variables $Z_{p}$ and $Z_{q}$ to the joint PDF of random variables $Z_{p-q}$ and $Z_{p+q}$

$$
f_{Z_{p-q} Z_{p+q} \mid C_{i}^{1}}\left(z_{p-q}, z_{p+q}\right)=\frac{1}{|\operatorname{det} \mathbf{A}|} f_{Z_{p} Z_{q} \mid C_{i}^{1}}\left(z_{p}, z_{q}\right)
$$

where $|\operatorname{det} \mathbf{A}|=\mathbf{2}$. Hence, $P\left(Z_{p} \in \mathcal{I}_{p}, Z_{q} \in \mathcal{I}_{q} \mid C_{i}^{1}\right)$ becomes

$$
\begin{aligned}
P & \left(Z_{p} \in \mathcal{I}_{p}, Z_{q} \in \mathcal{I}_{q} \mid C_{i}^{1}\right) \\
& =\int_{z_{p} \in \mathcal{I}_{p}} \int_{z_{q} \in \mathcal{I}_{q}} f_{Z_{p} Z_{q} \mid C_{i}^{1}}\left(z_{p}, z_{q}\right) d z_{p} d z_{q} \\
& =|\operatorname{det} \mathbf{A}| \int_{R_{A}} f_{Z_{p-q} Z_{p+q} \mid C_{i}^{1}}\left(z_{p-q}, z_{p+q}\right) d z_{p+q} d z_{p-q} .
\end{aligned}
$$

Since $Z_{p}$ and $Z_{q}$ conform to i.i.d. normal distributions, $Z_{p+q}$ and $Z_{p-q}$ are also normal distributions. Moreover, $Z_{p+q}$ and $Z_{p-q}$ are independent because

$$
\begin{aligned}
\operatorname{Cov}\left(Z_{p+q}, Z_{p-q}\right) & =\operatorname{Cov}\left(Z_{p}+Z_{q}, Z_{p}-Z_{q}\right) \\
& =\operatorname{Var}\left(Z_{p}\right)-\operatorname{Var}\left(Z_{q}\right)=0 .
\end{aligned}
$$

Therefore, we know

$$
\begin{aligned}
f_{Z_{p-q} \mid C_{i}^{1}}\left(z_{p-q}\right) & =\operatorname{Bel}\left(\mu_{p}-\mu_{q}, 2 \sigma^{2}\right) \\
f_{Z_{p+q} \mid C_{i}^{1}}\left(z_{p+q}\right) & =\operatorname{Bel}\left(\mu_{p}+\mu_{q}, 2 \sigma^{2}\right) \\
f_{Z_{p-q} Z_{p+q} \mid C_{i}^{1}}\left(z_{p-q}, z_{p+q}\right) & =f_{Z_{p+q} \mid C_{i}^{1}}\left(z_{p+q}\right) \\
f_{Z_{p-q} \mid C_{i}^{1}}\left(z_{p-q}\right) . &
\end{aligned}
$$

The integral over $R_{A}$ in (29) can be calculated as follows:

$$
\begin{aligned}
& \iint_{R_{A}} f_{Z_{p-q} Z_{p+q} \mid C_{i}^{1}}\left(z_{p-q}, z_{p+q}\right) d z_{p+q} d z_{p-q} \\
& =\int_{\tilde{z}_{p}-\tilde{z}_{q}-1}^{\tilde{z}_{p}-\tilde{z}_{q}+1}\left(f_{Z_{p-q} \mid C_{i}^{1}}\left(z_{p-q}\right)\right. \\
& \left.\quad \times \int_{2 \tilde{z}_{q}-1+z_{p-q}}^{2 \tilde{z}_{p}+1-z_{p-q}} f_{Z_{p+q} \mid C_{i}^{1}}\left(z_{p+q}\right) d z_{p+q}\right) d z_{p-q} .
\end{aligned}
$$

To simplify the above integral, let us define

$$
g\left(z_{p-q}\right)=\int_{2 \tilde{z}_{q}-1+z_{p-q}}^{2 \tilde{z}_{p}+1-z_{p-q}} f_{Z_{p+q} \mid C_{i}^{1}}\left(z_{p+q}\right) d z_{p+q} .
$$

Using the first mean value theorem for integration, we derive the relation between $P\left(Z_{p} \in \mathcal{I}_{p}, Z_{q} \in \mathcal{I}_{q} \mid C_{i}^{1}\right)$ and $P\left(Z_{p-q} \in\right.$ $\left.\mathcal{I}_{p-q} \mid C_{i}^{1}\right)$ as

$$
\begin{aligned}
& P\left(Z_{p} \in \mathcal{I}_{p}, Z_{q} \in \mathcal{I}_{q} \mid C_{i}^{1}\right) \\
& =|\operatorname{det} \mathbf{A}| \cdot \int_{\tilde{z}_{p}-\tilde{z}_{q}-1}^{\tilde{z}_{p}-\tilde{z}_{q}+1} f_{Z_{p-q} \mid C_{i}^{1}}\left(z_{p-q}\right) g\left(z_{p-q}\right) d z_{p-q} \\
& =2 g(\xi) \int_{\tilde{z}_{p}-\tilde{z}_{q}-1}^{\tilde{z}_{p}-\tilde{z}_{q}+1} f_{Z_{p-q} \mid C_{i}^{1}}\left(z_{p-q}\right) d z_{p-q} \\
& =2 g(\xi) P\left(Z_{p-q} \in \mathcal{I}_{p-q} \mid C_{i}^{1}\right) \\
& =\frac{1}{\eta_{p q}} P\left(Z_{p-q} \in \mathcal{I}_{p-q} \mid C_{i}^{1}\right)
\end{aligned}
$$

where $\xi \in \mathcal{I}_{p-q}$, and $\eta_{p q}=\frac{1}{2 g(\xi)}$ is the normalizing factor. This completes the proof.

\section{APPENDIX B}

\section{PROOF OF LEMMA 3}

Assume robot $q, q \in M_{0}$, has an ideal receiver which does not have the sensing range limit. Denote $Z_{q}=\psi_{q}+\omega_{q}$ as the RSS readings of the ideal receiver. The ideal receiver would allow us to use $Z_{p-q}^{11}$ as the RSS ratio instead of $Z_{p-q}^{10}$ from a regular receiver. In addition, $\psi_{q} \leq \zeta$. According to (4), we know that

$$
Z_{p-q}^{10}=Z_{p-q}^{11}+Z_{q}-\left(\zeta+\omega_{q}\right) .
$$


The PDF of $\left(Z_{p-q}^{10} \mid C_{i}^{1}\right)$ is rewritten by

$$
\begin{aligned}
& f_{Z_{p-q}^{10} \mid C_{i}^{1}}\left(z_{p-q}^{10}\right) \\
& =f_{Z_{p-q}^{10} \mid C_{i}^{1}, Z_{p}>Z_{q}}\left(z_{p-q}^{10}\right) P\left(Z_{p}>Z_{q}\right) \\
& \quad+f_{Z_{p-q}^{10} \mid C_{i}^{1}, Z_{p} \leq Z_{q}}\left(z_{p-q}^{10}\right) P\left(Z_{p} \leq Z_{q}\right) \\
& =f_{Z_{p-q}^{10} \mid C_{i}^{1}, Z_{p}>Z_{q}}\left(z_{p-q}^{10}\right)
\end{aligned}
$$

where $P\left(Z_{p}>Z_{q}\right)=1$, and $P\left(Z_{p} \leq Z_{q}\right)=0$.

Conditioning on $Z_{q}$ and using the first mean value theorem for integration, the PDF of $\left(Z_{p-q}^{10} \mid C_{i}^{1}, Z_{p}>Z_{q}\right)$ becomes

$$
\begin{aligned}
& f_{Z_{p-q}^{10} \mid C_{i}^{1}, Z_{p}>Z_{q}}\left(z_{p-q}^{10}\right) \\
& =\int_{-\infty}^{+\infty} f_{Z_{p-q}^{10}, Z_{q} \mid C_{i}^{1}, Z_{p}>Z_{q}}\left(z_{p-q}^{10}, z_{q}\right) d z_{q} \\
& =\int_{-\infty}^{\zeta+\omega_{q}} \frac{f_{Z_{p-q}^{10}, Z_{q} \mid C_{i}^{1}, Z_{p}>Z_{q}}\left(z_{p-q}^{10}, z_{q}\right)}{f_{Z_{q}} \mid C_{i}^{1}\left(z_{q}\right)} \cdot f_{Z_{q} \mid C_{i}^{1}}\left(z_{q}\right) d z_{q} \\
& =\int_{-\infty}^{\zeta+\omega_{q}} f_{Z_{p-q}^{10} \mid Z_{q}, C_{i}^{1}, Z_{p}>Z_{q}}\left(z_{p-q}^{10} \mid z_{q}\right) f_{Z_{q} \mid C_{i}^{1}}\left(z_{q}\right) d z_{q} \\
& =f_{Z_{q} \mid C_{i}^{1}}\left(\xi^{\prime}\right) \int_{-\infty}^{\zeta+\omega_{q}} f_{Z_{p-q}^{10} \mid Z_{q}, C_{i}^{1}, Z_{p}>Z_{q}}\left(z_{p-q}^{10} \mid z_{q}\right) d z_{q} \\
& =\frac{1}{\eta^{10}} \int_{-\infty}^{\zeta+\omega_{q}} f_{Z_{p-q}^{10} \mid Z_{q}, C_{i}^{1}, Z_{p}>Z_{q}}\left(z_{p-q}^{10} \mid z_{q}\right) d z_{q}
\end{aligned}
$$

where $-\infty \leq \xi^{\prime} \leq \zeta+\omega_{q}$, and $\eta^{10}=\frac{1}{f_{Z_{q} \mid C_{i}^{1}\left(\xi^{\prime}\right)}}$ is the normalizing factor.

Plugging (34) in, we have

$$
\begin{aligned}
& f_{Z_{p-q}^{10} \mid C_{i}^{1}, Z_{p}>Z_{q}}\left(z_{p-q}^{10}\right) \\
& =\frac{1}{\eta^{10}} \int_{-\infty}^{\zeta+\omega_{q}} f_{Z_{p-q}^{10} \mid Z_{q}, C_{i}^{1}, Z_{p}>Z_{q}}\left(z_{p-q}^{10} \mid z_{q}\right) d z_{q} \\
& =\frac{1}{\eta^{10}} \int_{z_{p-q}^{10}}^{+\infty} f_{Z_{p-q}^{11} \mid C_{i}^{1}}\left(z_{p-q}^{11}\right) d z_{p-q}^{11} \\
& =\frac{1}{\eta^{10}}\left(1-F_{Z_{p-q}^{11} \mid C_{i}^{1}}\left(z_{p-q}^{10}\right)\right) .
\end{aligned}
$$

Thus, we have

$$
\begin{aligned}
P & \left(Z_{p-q}^{10} \in \mathcal{I}_{p-q} \mid C_{i}^{1}\right) \\
& =\int_{\tilde{z}_{p}-\tilde{z}_{q}-1}^{\tilde{z}_{p}-\tilde{z}_{q}+1} f_{Z_{p-q}^{10} \mid C_{i}^{1}}(z) d z \\
& =\int_{\tilde{z}_{p}-\tilde{z}_{q}}^{\tilde{z}_{p}-\tilde{z}_{q}+1} f_{Z_{p-q}^{10} \mid C_{i}^{1}, Z_{p}>Z_{q}}(z) d z \\
& =\int_{\tilde{z}_{p}-\tilde{z}_{q}}^{\tilde{z}_{p}-\tilde{z}_{q}+1} \frac{1}{\eta^{10}}\left(1-F_{Z_{p-q}^{11} \mid C_{i}^{1}}(z)\right) d z \\
& =\frac{1}{\eta^{10}}\left(1-\int_{\tilde{z}_{p}-\tilde{z}_{q}}^{\tilde{z}_{p}-\tilde{z}_{q}+1} F_{Z_{p-q}^{11} \mid C_{i}^{1}}(z) d z\right) .
\end{aligned}
$$

This completes the proof.

\section{ACKNOWLEDGMENT}

The authors would like to thank E. Frew, E. Frazzoli, and J. Xiao for their insightful discussions. They would also thank D. Shell and B. Fine for sharing iRobot Create robots for the physical experiments. They also would like to thank for $\mathrm{W}$. $\mathrm{Li}$, Y. Lu, J. Lee, M. Hielsberg, S. Mun, M. Hirami, and S. Jacob for their inputs and contributions to the NetBot Laboratory at Texas A\&M University.

\section{REFERENCES}

[1] J. Letchner, D. Fox, and A. LaMarce, "Large-scale localization from wireless signal strength," in Proc. Nat. Conf. Artif. Intell., 2005, pp. 1520.

[2] N. Malhotra, M. Krasniewski, C. Yang, S. Bagchi, and W. Chappell, "Location estimation in ad hoc networks with directional antennas," in Proc. 25th IEEE Int. Conf. Distrib. Comput. Syst., Washington, DC, USA, 2005, pp. 633-642.

[3] G. Mao, B. Fidan, and B. Anderson, "Wireless sensor network localization techniques," Comput. Netw., vol. 51, no. 7, pp. 2529-2553, 2007.

[4] E. Nerurkar, S. Roumeliotis, and A. Martinelli, "Distributed maximum a posteriori estimation for multi-robot cooperative localization," in Proc. IEEE Int. Conf. Robot. Autom, May 2009, pp. 1402-1409.

[5] K. Lorincz and M. Welsh, "Motetrack: A robust, decentralized approach to RF-based location tracking," in Proc. Int. Workshop Location ContextAwareness Pervasive, 2005, pp. 63-82.

[6] D. Koutsonikolas, S. Das, and Y. Hu, "Path planning of mobile landmarks for localization in wireless sensor networks," Comput. Comunications, vol. 30, pp. 2577-2592, 2007.

[7] T. Sit, Z. Liu, M. Ang, and W. Seah, "Multi-robot mobility enhanced hopcount based localization in ad hoc networks," Robot. Autonomos Syst., vol. 55, pp. 244-252, 2007.

[8] B.-C. Liu, K.-H. Lin, and J.-C. Wu, "Analysis of hyperbolic and circular positioning algorithms using stationary signal-strength-difference measurements in wireless communications," IEEE Trans. Veh. Technol., vol. 55, no. 2, pp. 499-509, Mar. 2006.

[9] N. Patwari, I. Hero, A.O. M. Perkins, N. Correal, and R. O'Dea, "Relative location estimation in wireless sensor networks," IEEE Trans. Signal Process., vol. 51, no. 8, pp. 2137-2148, Aug. 2003.

[10] Y. Sun, J. Xiao, and F. Cabrera-Mora, "Robot localization and energyefficient wireless communications by multiple antennas," presented at the IEEE/RSJ Int. Conf. Intell. Robots Syst., St Louis, MO, USA, Oct. 2009.

[11] D. Li, K. Wong, Y. H. Hu, and A. Sayeed, "Detection, classification, and tracking of targets," IEEE Signal Process. Mag., vol. 19, no. 2, pp. 17-29, Mar. 2002.

[12] M. Kim and N. Y. Chong, "Direction sensing rfid reader for mobile robot navigation," IEEE Trans. Autom. Sci. Eng., vol. 6, no. 1, pp. 44-54, Jan. 2009.

[13] S. Thrun and Y. Liu, "Multi-robot slam with sparse extended information filers," in Robotics Research (ser. Springer Tracts in Advanced Robotics), P. Dario and R. Chatila, Eds. Berlin, Germany: Springer, 2005, vol. 15, pp. 254-266.

[14] A. Howard, "Multi-robot simultaneous localization and mapping using particle filters," Int. J. Robot. Res., vol. 25, no. 12, pp. 1243-1256, Dec. 2006.

[15] S. Carpin, "Fast and accurate map merging for multi-robot systems," Auton. Robots, vol. 25, pp. 305-316, Jul. 2008.

[16] W. Burgard, M. Moors, C. Stachniss, and F. Schneider, "Coordinated multi-robot exploration," IEEE Trans. Robot., vol. 21, no. 3, pp. 376-386, Jun. 2005.

[17] A. Elfes, "Using occupancy grids for mobile robot perception and navigation," Comput., vol. 22, pp. 46-57, Jun. 1989.

[18] H. Moravec, "Sensor fusion in certainty grids for mobile robots," AI Mag., vol. 9, pp. 61-74, 1988.

[19] S. Thrun, W. Burgard, and D. Fox, Probabilistic Robotics. Cambridge, MA, USA: MIT Press, 2005.

[20] Y. Altshuler, V. Yanovski, I. Wagner, and A. Bruckstein, "Multi-agent cooperative cleaning of expanding domains," Int. J. Robot. Res., vol. 30, no. 8, pp. 1037-1071, Aug. 2011.

[21] F. Bullo, J. Cortés, and S. Martínez, Distributed Control of Robotic Networks (ser. Applied Mathematics Series). Princeton, NJ, USA: Princeton Univ. Press, 2009. 
[22] W. Ren and Y. Cao, Distributed Coordination of Multi-Agent Networks (ser. Communications and Control Engineering Series). London, U.K.: Springer-Verlag, 2011.

[23] K. Zhou and S. Roumeliotis, "Optimal motion strategies for range-only constrained multi-sensor target tracking," IEEE Trans. Robot., vol. 24, no. 5, pp. 1168-1185, Oct. 2008.

[24] X. Zhou and S. Roumeliotis, "Robot-to-robot relative pose estimation from range measurements," IEEE Trans. Robot., vol. 24, no. 6, pp. 1379-1393, Dec. 2008.

[25] N. Trawny and S. Roumeliotis, "On the global optimum of planar, rangebased robot-to-robot relative pose estimation," in Proc. IEEE Int. Conf. Robot. Autom., Anchorage, AK, USA, May 2010, pp. 3200-3206.

[26] V. Huynh, J. Enright, and E. Frazzoli, "Persistent patrol with limited-range on-board sensors," in Proc. 49th IEEE Conf. Decision Control, Atlanta, GA, USA, Dec. 2010, pp. 7661-7668.

[27] D. Bhadauria, O. Tekdas, and V. Isler, "Robotic data mules for collecting data over sparse sensor fields," J. Field Robot., vol. 28, no. 3, pp. 388-404, 2011.

[28] D. Song, J. Yi, and Z. Goodwin, "Localization of unknown networked radio sources using a mobile robot with a directional antenna," in Proc. Amer. Control Conf., New York, NY, USA, Jul. 2007, pp. 5952-5957.

[29] D. Song, C. Kim, and J. Yi, "Simultaneous localization of multiple unknown CSMA-based wireless sensor network nodes using a mobile robot with a directional antenna," J. Intell. Service Robots, vol. 2, no. 4, pp. 219-233, Oct. 2009.

[30] D. Song, C. Kim, and J. Yi, "Monte carlo simultaneous localization of multiple unknown transient radio sources using a mobile robot with a directional antenna," presented at the IEEE Int. Conf. Robot. Autom., Kobe, Japan, May 2009.

[31] D. Song, C. Kim, and J. Yi, "Simultaneous localization of multiple unknown and transient radio sources using a mobile robot," IEEE Trans. Robot., vol. 28, no. 3, pp. 668-680, Jun. 2012.

[32] D. Song, C. Kim, and J. Yi, "Stochastic modeling of the expected time to search for an intermittent signal source under a limited sensing range," presented at the Robot., Sci. Syst. Conf., Zaragoza, Spain, Jun. 2010.

[33] D. Song, C. Kim, and J. Yi, "On the time to search for an intermittent signal source under a limited sensing range," IEEE Trans. Robot., vol. 27, no. 2, pp. 313-323, Apr. 2011.

[34] C. Kim, D. Song, and J. Yi, "Decentralized searching of multiple unknown and transient radio sources," presented at the IEEE Int. Conf. Robot. Autom., Karlsruhe, Germany, May 2013.

[35] C. Kim, D. Song, Y. Xu, and J. Yi, "Localization of multiple unknown transient radio sources using multiple paired mobile robots with limited sensing ranges," presented at the IEEE Int. Conf. Robot. Autom., Shanghai, China, May 2011.

[36] Z. Zaidi and B. Mark, "Real-time mobility tracking algorithms for cellular networks based on Kalman filtering," IEEE Trans. Mobile Comput., vol. 4, no. 2, pp. 195-208, Mar./Apr. 2005.

[37] J. A. Hartigan and M. Wong, "A k-means clustering algorithm," Appl. Statist., vol. 28 , pp. 100-108, 1979.

[38] M. Hahsler and K. Hornik, "TSP-Infrastructure for the traveling salesperson problem," J. Statist. Softw., vol. 23, no. 2, pp. 1-21, Dec. 2007.

[39] A. Leon-Garcia, Probability and Random Processes for Electrical Engineering, 2nd ed. Reading, MA, USA: Addison-Wesley, 1993.

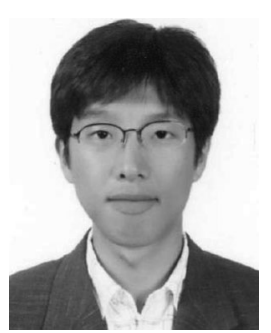

Chang-Young Kim (S'11-M'12) received the B.S. degree in electrical engineering from Korea University, Seoul, Korea, in 2006 and the Ph.D. degree from the Department of Computer Science and Engineering, Texas A\&M University, College Station, TX, USA, in 2012.

He is Robotics Software Engineer with Neato Robotics, Newark, CA, USA. He was involved in industry in the fields of automotive and embedded systems with GIT Co., Ltd., Korea. His research interests include mobile robots, networked robots, radio localization, robot navigation, sensor networks, and surveillance.

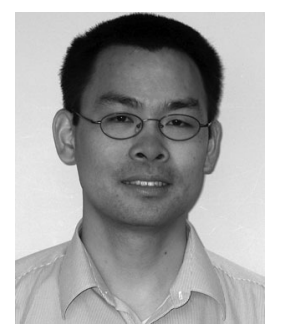

Dezhen Song (S'02-M'04-SM'09) received the B.S. and M.S. degrees from Zhejiang University, Zhejiang, China, in 1995 and 1998, respectively, and the Ph.D. degree from the University of California, Berkeley, CA, USA, in 2004.

$\mathrm{He}$ is an Associate Professor with Texas A\&M University, College Station, Texas, TX, USA. His primary research area is networked robotics, distributed sensing, computer vision, surveillance, and stochastic modeling.

Dr. Song received the Kayamori Best Paper Award from the 2005 IEEE International Conference on Robotics and Automation (with J. Yi and S. Ding). He received the NSF Faculty Early Career Development (CAREER) Award in 2007. From 2008 to 2012, he was an Associate Editor of the IEEE TRANSACTIONS ON ROBOTICS. He is currently an Associate Editor of the IEEE TRANSACTIONS ON AUTOMATION SCIENCE AND ENGINEERING.

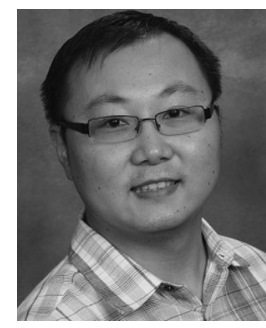

Yiliang Xu (S'05-M'11) received the B.E. degree in electrical engineering from Zhejiang University, Hangzhou, China, in 2002, the Ph.D. degree in electrical and electronic engineering from Nanyang Technological University, Singapore, in 2006, and another $\mathrm{Ph} . \mathrm{D}$. degree in computer science from Texas A\&M University, College Station, TX, USA, in 2011

$\mathrm{He}$ is currently a Research Engineer with Kitware Inc., Clifton Park, NY, USA. His research interests include networked robotics, computer vision, surveillance systems, and computational intelligence.

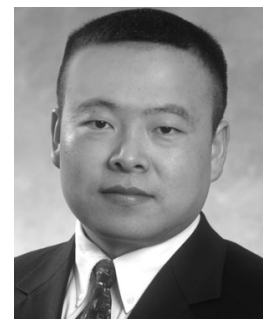

Jingang Yi (S'99-M'02-SM'07) received the B.S degree in electrical engineering from Zhejiang University, Hangzhou, China, in 1993, the M.Eng. degree in precision instruments from Tsinghua University, Beijing, China, in 1996, and the M.A. degree in mathematics and the Ph.D. degree in mechanical engineering from the University of California, Berkeley, CA, USA, in 2001 and 2002, respectively.

$\mathrm{He}$ is currently an Associate Professor of mechanical engineering with Rutgers University. His research interests include autonomous robotic systems, dynamic systems and control, mechatronics, and automation science and engineering, with applications to biomedical systems, civil infrastructure, and transportation systems.

Dr. Yi received the 2010 U.S. National Science Foundation (NSF) CAREER Award. He has coauthored papers that have received several best papers at the IEEE/ASME AIM, ASME DSCC, IEEE ICRA, etc. He currently serves as an Associate Editor for the IEEE TRANSACTIONS ON AUTOMATION SCIENCE AND ENGINEERING and several other journals for the ASME and IFAC.

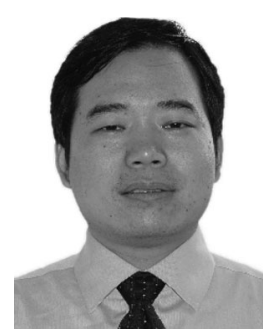

Xinyu Wu received the B.S. and M.S. degrees from the University of Science and Technology of China, Hefei, China, in 2001 and 2004, respectively, and the $\mathrm{Ph} . \mathrm{D}$. degree from the Chinese University of Hong Kong, Hong Kong, in 2008.

He is currently a Professor with the Shenzhen Institutes of Advanced Technology, Shenzhen, China, and an Associate Director with the Center for Intelligent and Biomimetic Systems. His research interests include computer vision, robotics, and intelligent systems. 